\title{
The zCOSMOS redshift survey: how group environment alters global downsizing trends ${ }^{\star}$
}

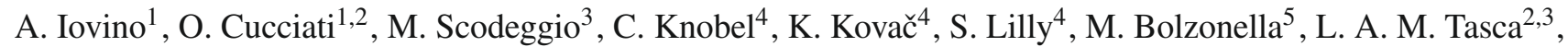 \\ G. Zamorani ${ }^{5}$, E. Zucca ${ }^{5}$, K. Caputi ${ }^{4}$, L. Pozzetti ${ }^{5}$, P. Oesch ${ }^{4}$, F. Lamareille ${ }^{6}$, C. Halliday ${ }^{7}$, S. Bardelli ${ }^{5}$,

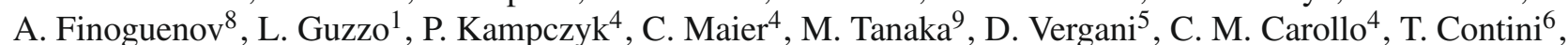 \\ J.-P. Kneib ${ }^{2}$, O. Le Fèvre ${ }^{2}$, V. Mainieri' ${ }^{9}$, A. Renzini ${ }^{10}$, A. Bongiorno ${ }^{8}$, G. Coppa ${ }^{5}$, S. de la Torre ${ }^{2,1}$, L. de Ravel ${ }^{2}$, \\ P. Franzetti ${ }^{3}$, B. Garilli ${ }^{3}$, J.-F. Le Borgne ${ }^{6}$, V. Le Brun ${ }^{2}$, M. Mignoli ${ }^{5}$, R. Pellò ${ }^{6}$, Y. Peng ${ }^{4}$, E. Perez-Montero ${ }^{6}$, \\ E. Ricciardelli ${ }^{10}$, J. D. Silverman ${ }^{4}$, L. Tresse ${ }^{2}$, U. Abbas ${ }^{11}$, D. Bottini ${ }^{3}$, A. Cappi ${ }^{5}$, P. Cassata ${ }^{2,12}$, A. Cimatti ${ }^{13}$, \\ A. M. Koekemoer ${ }^{15}$, A. Leauthaud ${ }^{14}$, D. Maccagni ${ }^{3}$, C. Marinoni ${ }^{16}$, H. J. McCracken ${ }^{17}$, P. Memeo ${ }^{3}$, B. Meneux ${ }^{8,18}$, \\ C. Porciani ${ }^{4,19}$, R. Scaramella ${ }^{20}$, D. Schiminovich ${ }^{21}$, and N. Scoville ${ }^{22}$ \\ (Affiliations can be found after the references)
}

Received 22 May 2009 / Accepted 8 October 2009

\section{ABSTRACT}

Context. Groups of galaxies are a common environment, bridging the gap between starforming field galaxies and quiescent cluster galaxies. Within groups secular processes could be at play, contributing to the observed strong decrease of star formation with cosmic time in the global galaxy population.

Aims. We took advantage of the wealth of information provided by the first $\sim 10000$ galaxies of the zCOSMOS-bright survey and its group catalogue to study in detail the complex interplay between group environment and galaxy properties.

Methods. The classical indicator $F_{\text {blue }}$, i.e., the fraction of blue galaxies, proved to be a simple but powerful diagnostic tool. We studied its variation for different luminosity and mass selected galaxy samples, divided as to define groups/field/isolated galaxy subsamples.

Results. Using rest-frame evolving $B$-band volume-limited samples, the groups galaxy population exhibits significant blueing as redshift increases, but maintains a systematic difference (a lower $F_{\text {blue }}$ ) with respect to the global galaxy population, and an even larger difference with respect to the isolated galaxy population. However moving to mass selected samples it becomes apparent that such differences are largely due to the biased view imposed by the $B$-band luminosity selection, being driven by the population of lower mass, bright blue galaxies for which we miss the redder, equally low mass, counterparts. By carefully focusing the analysis on narrow mass bins such that mass segregation becomes negligible we find that only for the lowest mass bin explored, i.e., $\log \left(\mathcal{M}_{*} / \mathcal{M}_{\odot}\right) \leq 10.6$, does a significant residual difference in color remain as a function of environment, while this difference becomes negligible toward higher masses.

Conclusions. Our results indicate that red galaxies of mass $\log \left(\mathcal{M}_{*} / \mathcal{M}_{\odot}\right) \geq 10.8$ are already in place at $z \sim 1$ and do not exhibit any strong environmental dependence, possibly originating from so-called nature or internal mechanisms. In contrast, for lower galaxy masses and redshifts lower than $z \sim 1$, we observe the emergence in groups of a population of nurture red galaxies: slightly deviating from the trend of the downsizing scenario followed by the global galaxy population, and more so with cosmic time. These galaxies exhibit signatures of group-related secular physical mechanisms directly influencing galaxy evolution. Our analysis implies that these mechanisms begin to significantly influence galaxy evolution after $z \sim 1$, a redshift corresponding to the emergence of structures in which these mechanisms take place.

Key words. galaxies: clusters: general - galaxies: evolution - galaxies: interactions

\section{Introduction}

Groups and clusters are commonly viewed as sites where environmental influences can affect the colors, star formation histories and morphologies of their member galaxies. One of the first pieces of empirical evidence supporting this claim was the observation by Butcher \& Oemler that clusters of galaxies contain a higher fraction of blue galaxies at progressively higher redshift, the so-called Butcher-Oemler effect (Butcher \& Oemler 1978, 1984). Their result provided direct observational evidence of strong, rapidly evolving galaxy population colors inside cluster cores with redshift.

Since these early papers, the Butcher-Oemler effect has been confirmed photometrically (Rakos \& Schombert 1995; Margoniner \& de Carvalho 2000; Margoniner et al. 2001; Kodama \& Bower 2001; Goto et al. 2003), spectroscopically

^ based on data obtained with the European Southern Observatory Very Large Telescope, Paranal, Chile, program 175.A-0839, PI: S. Lilly.
(Dressler \& Gunn 1982, 1992; Lavery \& Henry 1986, 1988; Fabricant et al. 1991; Poggianti et al. 1999, 2006; Ellingson et al. 2001), has been extended to groups (Allington-Smith et al. 1993; Wilman et al. 2005a; Gerke et al. 2007; Cucciati et al. 2009 b), and critically discussed in the context of selection biases (Andreon \& Ettori 1999; Andreon et al. 2004, 2006).

In parallel to these studies, evidence has emerged that the Universe as a whole formed stars more actively in the past than today (Lilly et al. 1996; Madau et al. 1998; Hopkins 2004; Schiminovich et al. 2005) and that the typical mass of galaxies where the bulk of star formation occurs is higher in the past than today, the so-called downsizing effect (Cowie et al. 1996; Gavazzi et al. 1996).

These observations questioned whether the Butcher-Oemler phenomenon is caused by physical mechanisms typical of dense environments, that significantly alter global trends displayed by the global galaxy population in the coeval field, or reflects the evolution of the global galaxy population. Interestingly, with 
growing evidence that denser environments only suppress star formation (Balogh et al. 2004b,a), we have started to test in groups and/or clusters at higher redshifts whether we measure a higher fraction of blue galaxies that is nevertheless lower than the coeval field.

Since groups contain a large fraction of galaxies in the nearby Universe, nearly 50\% (Huchra \& Geller 1982; Eke et al. 2004; Berlind et al. 2006), while only a few percent of galaxies are contained in the denser cluster cores, group-related transformations may drive the observed strong decrease in star formation with cosmic time, at least since $z \sim 1$, when these structures started to become predominant according to the hierarchical structure scenarios.

In the cores of rich clusters phenomena such as ram pressure stripping have been widely documented in the literature, as for well studied galaxies in the Virgo cluster, (Kenney et al. 2004; Vollmer et al. 2004), and observed in simulations (Brüggen \& De Lucia 2008).

In contrast, similar environment-dependent effects in groups are less clearly defined, although possibilities have been presented in the literature, including gradual cessation of star formation induced either by gentle gas stripping and starvation by a diffuse intragroup medium, or by slow group-scale harassment (Larson et al. 1980; Moore et al. 1999; Gnedin 2003; Roediger \& Hensler 2005; Kawata \& Mulchaey 2008).

From a theoretical perspective, numerical simulations incorporating the standard cosmological paradigm suggest that galaxy properties such as, e.g., colors, spin etc.) are primarily determined by the mass of the dark matter halo in which the galaxy resides (Cooray \& Sheth 2002), and that, at a given mass, in overdense environments dark matter haloes assemble at higher redshifts than in underdense environments (Gao et al. 2005). This framework could provide a simple way of explaining the observed trends in colors with galaxy luminosity, mass and environment, at low (De Propris et al. 2004; Blanton \& Berlind 2007) and also at high redshifts (Wilman et al. 2005a; Balogh et al. 2007), without resorting to any specific mechanisms acting in groups. Two large recent redshift surveys, VVDS and DEEP2, have addressed this problem by studying both groups (Gerke et al. 2007; Cucciati et al. 2009b) and local density field measurements (Cucciati et al. 2006; Cooper et al. 2006, 2007), although both studies considered both luminosity selected samples, a choice that, as we discuss later, offers only partial insight into the problem.

The question of which variables are needed to fully define galaxy evolution therefore remains unanswered, and is usually considered in terms of either nature or nurture processes. This corresponds to asking whether: galaxy evolution is driven mainly by internal processes, imprinted at galaxy birth, that operate inside the average galaxy, or group environment has a specific effect on shaping galaxy evolution, because of specific mechanisms taking place in dense, possibly virialized regions, where secular influences have better chances to affect galaxy evolution.

To distinguish between the effects of environment and trends related to galaxy evolution with redshift, one needs homogeneous and sizeable group and field galaxy samples, covering a wide redshift range and with reliable measurements of galaxy rest-frame colors, luminosities and masses. These data would allow us to monitor with look-back time the evolutionary histories of galaxies located in different group/field environments, and to disentangle between the different dependencies and their relative importance.
The advantage of the data-set used in our analysis is that it satisfies all of these requirements. zCOSMOS is a survey tailored for studying the large scale structure and detecting groups up to $z \sim 1$ (Lilly et al. 2007, 2009). Its large volume coverage and small errors in galaxy redshift measurements enable the production, even for the first batch of $\sim 10000$ measured redshifts, of a large group catalogue containing 102 groups with $N \geq 5$ spectroscopically confirmed members and a further $\sim 700$ going down to pairs (Knobel et al. 2009). Furthermore this catalogue, because of the precise fine tuning of the algorithm used for group detection, is remarkably free from contamination and incompleteness, especially at the low richness, low velocity dispersion end and, most importantly, its quality is stable as a function of redshift (Knobel et al. 2009). Last but not least, the large amount of precise photometric ancillary data available from the COSMOS survey (Scoville et al. 2007), provides robust estimates of the fundamental properties of each galaxy, such as restframe luminosities, colors and masses. We are therefore in best position with our data-set to investigate in detail which processes are the most influential in shaping galaxy evolution.

Complementary analyses of the same 10K-sample data-set have been carried out in other papers. Kovac et al. (2009b) in a parallel paper study the influence of group environment in shaping galaxy morphologies. Using the density field measured in Kovač et al. (2009a), Zucca et al. (2009) and Bolzonella et al. (2009) study the galaxy luminosity and mass functions respectively as a function of environment, while Cucciati et al. (2009a) and Tasca et al. (2009) investigate the dependencies of galaxy colors and morphologies, respectively, from the general density field. Finally Silverman et al. (2009) and Vergani et al. (2010) study how environment plays a role in triggering active galactic nuclei activity and in quenching star-formation respectively. For more details, we refer the interested reader to those papers.

A concordance cosmology is adopted throughout our paper, with $h_{70}=H_{0} / 70 \mathrm{~km} \mathrm{~s}^{-1} \mathrm{Mpc}^{-1}, \Omega_{\mathrm{m}}=0.25$ and $\Omega_{\Lambda}=0.75$. All magnitudes are always quoted in the AB system.

\section{Samples used in the analysis}

\subsection{The zCOSMOS 10K-sample}

The zCOSMOS survey is a large spectroscopic survey undertaken in the COSMOS field (Scoville et al. 2007), using $600 \mathrm{~h}$ of observations with the VIMOS spectrograph at VLT. It consists of two parts: zCOSMOS-bright and zCOSMOS-deep. zCOSMOSbright is a survey purely magnitude limited in I-band; when complete it will provide a sample of $\sim 20000$ galaxies in the range $15.0 \leq I_{A B} \leq 22.5$ from the HST ACS imaging (Koekemoer et al. 2007) over the whole area of $1.7 \mathrm{deg}^{2}$ of the COSMOS field. zCOSMOS-deep targets $\sim 10000$ galaxies, selected through color criteria to have $1.4 \lesssim z \lesssim 3.0$ within the central $1 \mathrm{deg}^{2}$. At completion it will provide redshifts for $\sim 10000$ galaxies with magnitudes $B_{A B} \leq 25.0$.

The analysis presented in this paper uses of the sample of 10644 objects for which we obtained spectra during the first half of the zCOSMOS-bright observational campaign. This number corresponds to a total of 83 pointings of the VIMOS spectrograph, observed during ESO periods P75, P76, and P77 and includes compulsory, i.e., objects with forced slit positioning, and secondary targets, i.e., objects serendipitously falling inside the slit other than the primary target.

zCOSMOS-bright observations use the $R=600 \mathrm{MR}$ grism and $1 \mathrm{~h}$ integrations to secure redshifts with a high success rate. The wavelength range covered goes from $5500 \leq \lambda \leq 9700 \AA$. 

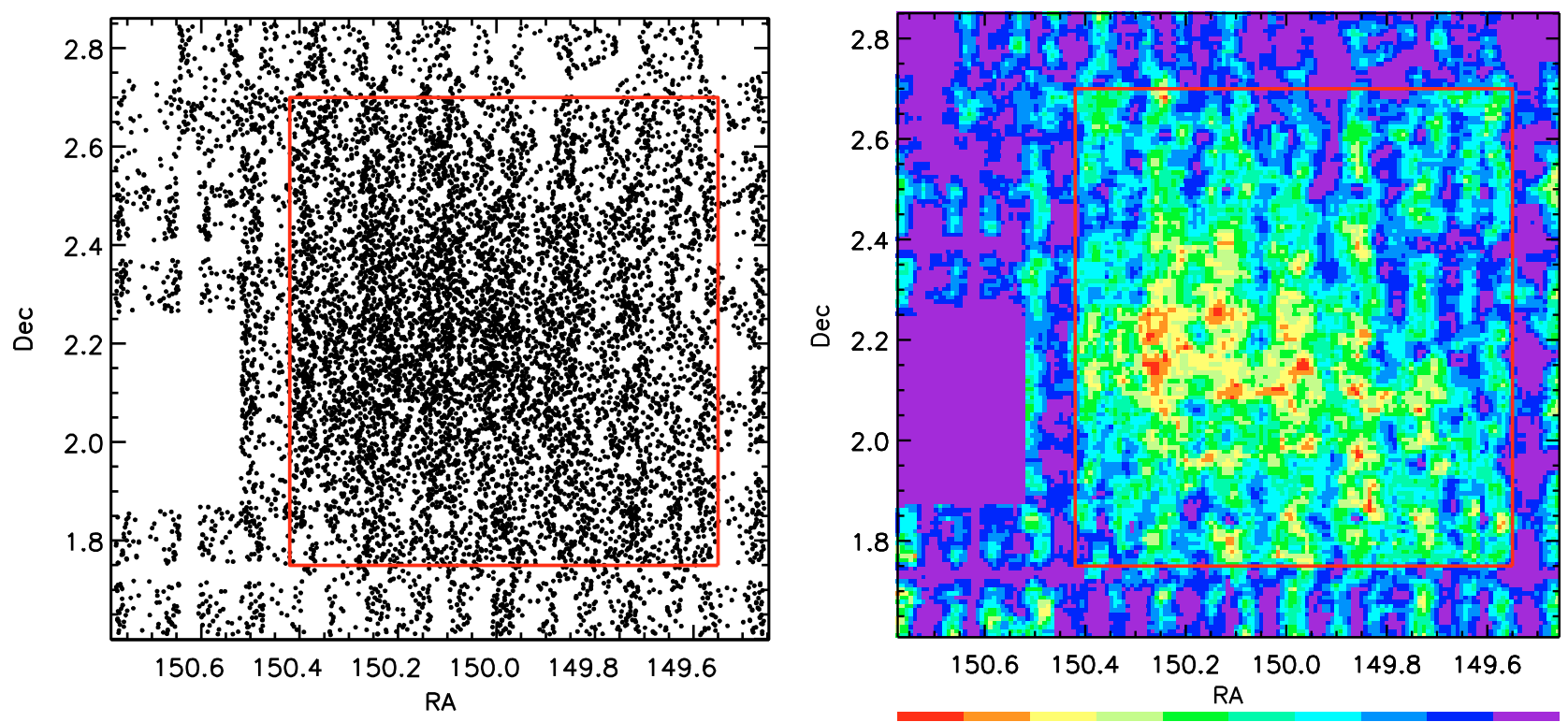

Fig. 1. The left panel shows the Ra - Dec distribution of the $\sim 10000$ objects observed in the first half of the zCOSMOS-bright survey. The right panel shows the $\mathrm{Ra}$ - Dec distribution of the ratio of the number of objects with reliable spectroscopic redshift to the total number of potential targets i.e., non-stellar objects in the parent bright photometric catalogue $\left(15.0 \leq I_{A B} \leq 22.5\right)$. The color scale provides the legenda for the range of values displayed on the plot. In both panels the red rectangle indicates the restricted area used for the analysis presented in this paper, corresponding to $\sim 0.83$ sq. deg. Within this restricted area the mean value of the sampling rate is around $40 \%$, i.e., two objects out of five have a reliable redshift measured (see text for more details).

For more details of the survey strategy and characteristics we refer the reader to Lilly et al. (2007) and Lilly et al. (2009).

The distribution on the sky of the $\sim 10000$ objects observed in the first half of the zCOSMOS-bright survey is illustrated in the left panel of Fig. 1. The vertical banding visible in the external, less finely sampled, regions reflects the quadrant design of VIMOS and an additional pattern introduced by the slit positioning software SPOC (Bottini et al. 2005). This pattern should almost completely disappear at survey completion, since the observational strategy foresees an eight-pass coverage with two mask designs at each pointing. The expected final sampling rate is around $\sim 70 \%$.

The redshift distribution of the observed galaxies covers the range $0.1 \leq z \leq 1.2$ and peaks at redshift $\sim 0.7$. The error in the redshift measurement, as determined from repeated observations, is around $100 \mathrm{~km} \mathrm{~s}^{-1}$, an accuracy well suited to the original survey scientific goals of the investigation of large scale structure and the detection of groups.

For each measured redshift, we adopted a ranking scheme reflecting our confidence in its correctness. It is based on six broad confidence classes (0-1-2-3-4-9) reflecting the quality of the redshift measurement as obtained from the spectra. This scheme is similar to that originally adopted in the CFRS (Le Fevre et al. 1995) and VVDS (Le Fèvre et al. 2005), but with some further refinements taking advantage of the wealth of photometric information available for each targeted object.

The large, exquisite quality, ancillary photometric database provided by the COSMOS survey (from HST data, to Spitzer, Galex, Chandra, CFHTLS, and Subaru data, see Scoville et al. 2007) has enabled us to derive reliable photometric redshifts for all objects in the zCOSMOS-bright parent photometric catalogue, with an uncertainty as low as $\Delta z \sim 0.01 \times(1+z)$ (Ilbert et al. 2009).

The photometric redshift information was used to incorporate in our analysis objects whose spectroscopic redshift, although less secure, was consistent with its photometric value and therefore deemed reliable $(\delta z$ smaller that $0.08 \times(1+z)$, see Lilly et al. 2009, for more details). In this way one can use $\sim 85 \%$ of the observed sample, totalling $\sim 8600$ galaxies up to $z=2.0$ ( 9200 including stars and with no high redshift cut-off), with a nominal spectroscopic confirmation rate of $\sim 98.5 \%$ as found by duplicate observations. This sample represents roughly half of the final zCOSMOS sample and when we talk of the 10K-sample we always refer to this subset of objects, the same used to perform group searches (Knobel et al. 2009).

For the 10K-sample galaxies, absolute rest-frame magnitudes and stellar masses were obtained using standard multicolor spectral energy distribution (SED) fitting analysis. Restframe absolute magnitudes were obtained using the ZEBRA code, for which a detailed description is provided in Feldmann et al. (2006) and Oesch et al. (in prep.). We note here that the templates used by ZEBRA are the standard CWW templates (Coleman et al. 1980) and starburst templates from Kinney et al. (1996), and the best fit template is normalized to each galaxy photometry and spectroscopic redshift.

Stellar masses in units of solar masses were obtained by fitting stellar population synthesis models to the multicolor spectral SED of the observed magnitudes using the Hyperzmass code (Bolzonella et al. 2009; Pozzetti et al. 2009). In the subsequent analysis, we use stellar masses calculated adopting the Bruzual \& Charlot (2003) libraries, and assuming a Chabrier initial mass function (Chabrier 2003). More details about the Hyperzmass code can be found in Bolzonella et al. (2009).

Panel (a) of Fig. 2 shows the distribution along the line of sight of the galaxies within the boundaries defined by $149.55 \leq$ $\mathrm{Ra} \leq 150.42,1.75 \leq \mathrm{Dec} \leq 2.70$ and $0.1 \leq z \leq 1.0$. These boundaries (see Sect. 3.1) are those adopted in our analysis to avoid being affected by the inhomogeneous coverage of the $10 \mathrm{~K}$-sample. The number of galaxies surviving within these $\mathrm{Ra}-\mathrm{Dec}-z$ boundaries equals 6204, of which 1966 are in groups, while 1146 define the so-called isolated galaxy sample (see Sect. 2.3). 


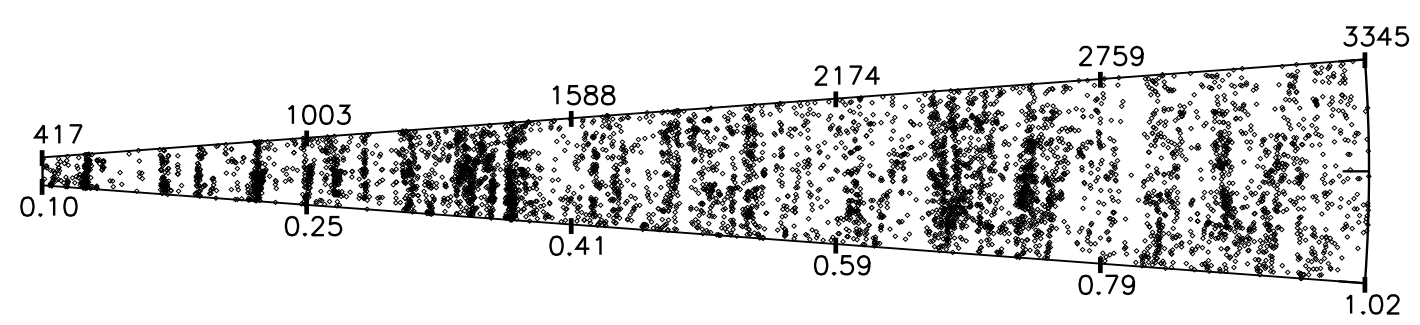

(a)
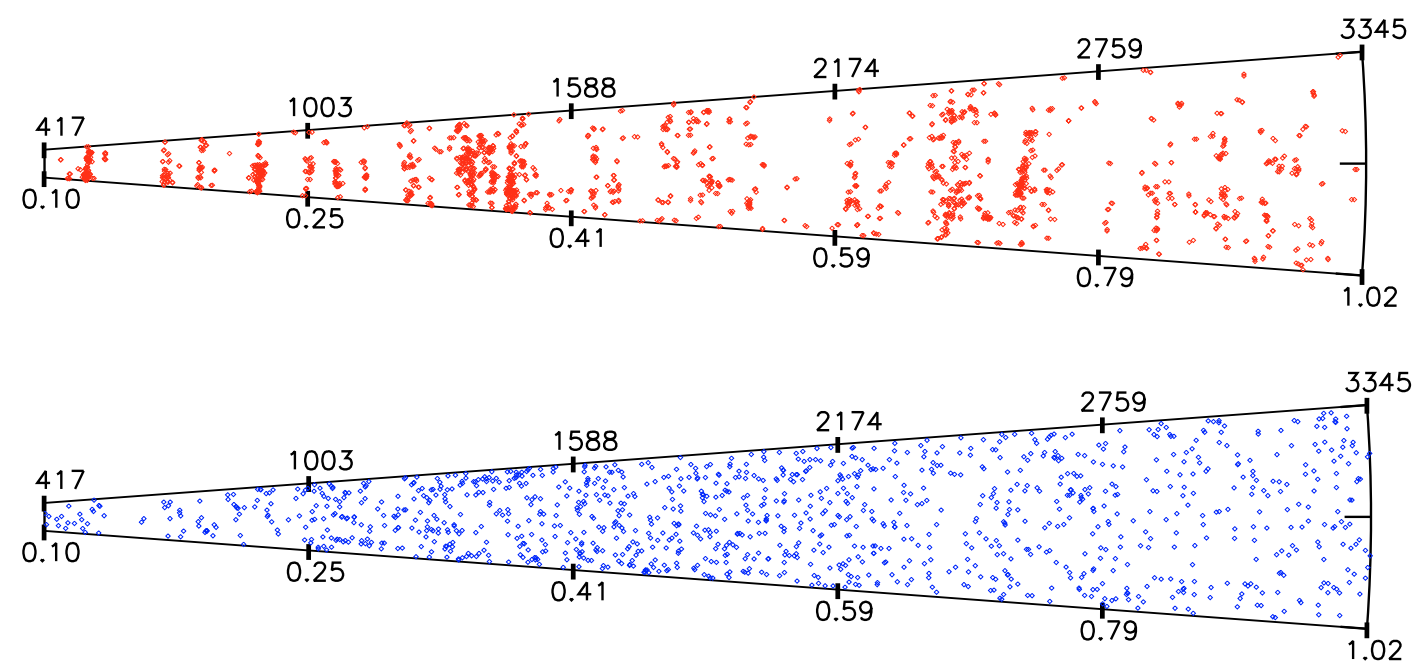

(c)

Fig. 2. Panel a) shows the distribution along the line of sight of the 6204 galaxies used in our analysis, the subset of the 10K-sample galaxies sample located within the central, high sampling rate, region of the survey and in the redshift range $0.1 \leq z \leq 1.0$. Panel b) shows the distribution along the line of sight of the 1966 group galaxies, while panel c) show the same for the sample of 1146 isolated galaxies. In each panel we collapsed the Dec axis and expanded by a factor of $\times 10$ the distances corresponding to the Ra axis to make the plot easier to read. Labels on the top of the cone indicate the line-of-sight distance in units of comoving $h_{70}^{-1} \mathrm{Mpc}$, while those on the bottom indicates the redshift. The transverse dimension at redshift $z=0.1$ is $\sim 7 h_{70}^{-1} \mathrm{Mpc}$, while that at redshift $z=1.0$ is $\sim 50 h_{70}^{-1} \mathrm{Mpc}$, corresponding to the dimension of $\Delta \mathrm{Ra} \sim 0.87 \mathrm{deg}$ of our restricted sample.

The transverse dimension of this restricted sample along Ra is $\sim 7 h_{70}^{-1} \mathrm{Mpc}$ at $z=0.1$, and $\sim 50 h_{70}^{-1} \mathrm{Mpc}$ at $z=1.0$. In the same redshift interval the total contiguous comoving volume sampled is $\sim 3.3 \times 10^{6} h_{70}^{-1} \mathrm{Mpc}^{3}$.

\subsection{The $10 \mathrm{~K}$ group catalogue}

In our analysis, we use the catalogue of groups presented in Knobel et al. (2009) and refer the reader to that paper for a detailed presentation of both the group finding algorithm and the group catalogue. Here we summarize the main points and advantages of the adopted group finding algorithm and briefly discuss the resulting group catalogue. Knobel et al. (2009) introduced a novel method, defined as a "multi-pass procedure", to achieve an impressive quality in group reconstruction as tested using realistic mock catalogues. This method, when combined with the standard fried-of-friends (FOF) algorithm, yields values of completeness and purity for the group catalogue obtained that are extremely stable with both redshift and number of members observed in the reconstructed groups. Typical values of these two quantities for groups reconstructed with more than five observed members are around $\sim 80 \%$ at all redshifts and do not decrease substantially for groups with lower number of members. Correspondingly the interloper fraction always remains below $\sim 20 \%$ at all redshifts for groups reconstructed with more than five observed members, with only a slight increase for groups with lower number of members (Knobel et al. 2009).
These results provide reassurance that the group catalogue that we use in our subsequent analysis is highly homogeneous up to $z \sim 1$, a fundamental prerequisite, since the aim of this paper is to explore redshift trends in group galaxy colors. If our results are to be reliable, we need to be confident that the group catalogue we use is almost entirely free from redshift dependent biases. The presence of a significantly higher interloper fraction with increasing redshift could surreptitiously increase the fraction of blue (field) galaxies observed in our group catalogue and be mistakenly interpreted as evidence of evolution. The extensive tests performed in Knobel et al. (2009) place on solid basis the analysis that we perform in the following sections.

Panel (b) of Fig. 2 shows the distribution along the line of sight of the group galaxy population (1966 galaxies in total) within the boundaries defined by $149.55 \leq \mathrm{Ra} \leq 150.42$, $1.75 \leq$ Dec $\leq 2.70$ and $0.1 \leq z \leq 1.0$. The presence of large structures is clearly delineated by the group galaxy sample. In fact there are quite a few conspicuous structures visible in this plot, e.g., those around redshifts $\sim 0.35$ and $\sim 0.7$, while there are, on the other hand, regions devoid of large structures, e.g., in the redshift range $0.4 \leq z \leq 0.6$ (see Kovač et al. 2009a, for a detailed description of the density field structures in the 10K-sample field).

We also note that our survey does not contain any single rich cluster, for example comparable to Coma cluster in the local Universe. This is not unexpected: because the size of the volume of the Universe explored by zCOSMOS-bright the 
probability of one such cluster being observed is negligible (see also Finoguenov et al. 2007).

\subsection{The isolated galaxy sample}

We complemented the analysis performed on the sample of group galaxies with a parallel one on a sample of isolated galaxies, i.e., a sample of galaxies located in low density regions. This comparative analysis should highlight the differences - if any in properties (rest frame colors in our analysis) from the group galaxy sample and therefore allow us to quantify the environmental dependencies of the properties explored more reliably.

To define the isolated galaxy sample, we use the Voronoi Tessellation method (Voronoi 1908). Voronoi Tessellation divides the space occupied by the survey into a set of unique polyhedral sub-volumes, each containing exactly one galaxy and all points in space that are closer to that galaxy than to any other. As a consequence, while galaxies with many neighbors (e.g., those in groups and high density environments) have small Voronoi volumes, relatively isolated galaxies have larger Voronoi volumes. Voronoi Tessellation has been used in the literature as a basis for group-finding algorithms (Marinoni et al. 2002; Gerke et al. 2005; Cucciati et al. 2009b; Knobel et al. 2009). It is quite straightforward to use Voronoi volumes to select a sample of isolated galaxies, defined as galaxies occupying the largest Voronoi volumes. This strategy has the advantage of being non-parametric, i.e., it avoids any arbitrarily chosen smoothing/window profile in defining low density regions.

However, proper attention must be taken to exclude galaxies that are close to survey borders and correct for the progressive increase in the typical size of Voronoi volumes between low and high redshifts in our flux-limited galaxy sample.

To avoid the first problem, i.e., of galaxies near the survey boundaries entering the isolated galaxy sample because of their apparently large Voronoi volumes, we decided to restrict the volume of the search for isolated galaxies within the boundaries defined by $149.57 \leq \mathrm{Ra} \leq 150.41,1.76 \leq \mathrm{Dec} \leq 2.68$ and $0.1 \leq z \leq 1.0$, which is slightly more restrictive than the limits adopted for the group analysis indicated by the red lines in Fig. 1. Furthermore, in all the subsequent analysis we decided to reject all isolated galaxies located in areas of lower sampling, that is galaxies with mean correction factor $\psi(\alpha, \delta) \geq 5$ (see Sect. 3.1). For these galaxies, a large measured Voronoi volume could be the result of the low spectroscopic sampling rate in the surrounding area.

To avoid the second possible problem, of being biased in the definition of isolated galaxies by the progressive decrease in the galaxy density in our flux-limited sample, we computed the median value of the logarithm of Voronoi volumes sizes as a function of redshift using running bins of size $\Delta z \leq 0.2$ in redshift steps of 0.05 . A simple linear fit to this quantity (as deemed reasonable by visual inspection) was then used to normalize all measured Voronoi volumes, correcting for the progressive increase with redshift in the mean inter-galaxy separation. We then selected the highest quartile of the normalized volumes distribution obtained in this way, after taking the simple precaution of further rejecting galaxies (148 in total, 80 at $z \leq 0.25$ ) whose normalized Voronoi volume was more than 100 times larger than the median one: mostly galaxies located too close to the survey borders, as suggested by the large predominance of low redshift objects and by their general distribution on the sky. Figure 3 illustrates the method adopted to select isolated galaxies. The final number of isolated galaxies obtained this way is 1146, after

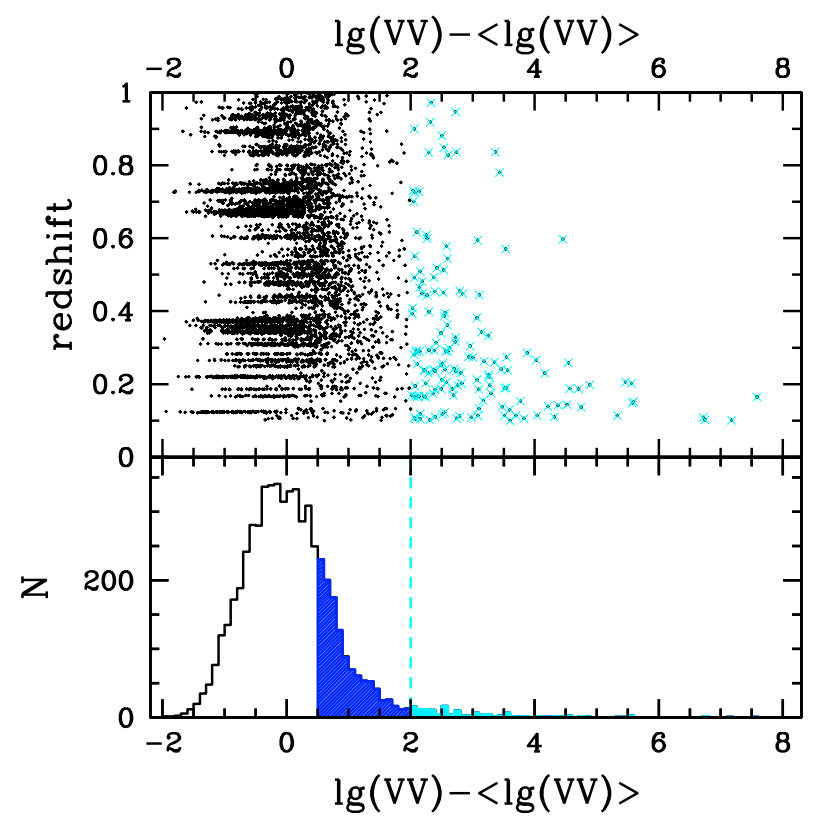

Fig. 3. Top panel: distribution of logarithm of normalized Voronoi volumes as a function of redshift. The stripes extending towards lower Voronoi volumes values are due to the presence of groups. Points marked with a cyan cross correspond to galaxies removed from the isolated galaxy sample because their Voronoi volume exceeded by a factor of 100 that of the median at the corresponding redshift. Bottom panel: histogram of the total distribution of normalized Voronoi volumes. The shaded blue area corresponds to the last quartile of the distribution, chosen to select our isolated galaxies sample, while the long tail in cyan extending to higher values - and indicated also by the vertical line - is the one clipped from the sample.

the removal of galaxies (206, out of which 128 located in pairs) listed in our group catalogue.

We checked the reliability of our approach by selecting isolated galaxies in simulations. We used the 24 COSMOS mock light-cones kindly provided by Kitzbichler, (Kitzbichler $\&$ White 2007), based on the Millennium DM N-body simulations (Springel 2005). We applied the same observational strategy to these cones used to select the 10K-sample: we chose the same pointings observed in $10 \mathrm{~K}$-sample, used SPOC to select observed targets and included the same redshift success rate as the real data. Out of the sample of isolated galaxies obtained from the mocks using the procedure described above, $\sim 60 \%$ are truly isolated galaxies, with a variance of a few percent from cone to cone, i.e., galaxies that in mock light-cones are inside a halo that contains only one galaxy down to the $R=26$ mag limit. However, when considering the $10 \mathrm{~K}$ mock samples - limited to $I_{A B}=22.5$ and with our sampling rate applied - this number increases to $\sim 90 \%$. In other words, only $\sim 10 \%$ of the galaxies in the isolated sample selected using our strategy are located in groups with at least two members in the $10 \mathrm{~K}$ mock samples. We should get rid of most of this contamination by the last step of our procedure: the final trimming of galaxies listed in our real group catalogue.

Panel (c) of Fig. 2 shows the distribution along the line of sight of the isolated galaxy sample, whose uniformity is evident.

\section{Measuring $\boldsymbol{F}_{\text {blue }}$}

We use in this paper the diagnostic tool introduced in the literature in the seminal work by Butcher \& Oemler (1978). These 
authors were the first to note that the fraction of blue galaxies ( $F_{\text {blue }}$ from now onwards) in clusters seems to increase with redshift. Their work started a long-lasting wave of observational and theoretical papers, which is still far from being completed (see the short literature review presented in the introduction).

After thirty years, the value of $F_{\text {blue }}$ is still a valuable and effective empirical tool in studying of the dependence of galaxy evolution from the environment in which they reside.

Galaxy color is the easiest parameter to measure among those that exhibit a distinctive bi-modality: spectral class, morphology, star formation rates and metallicities (see Strateva et al. 2001; Mignoli et al. 2009). Therefore it is the simplest to adopt in parametrizing the differences between evolution of groups and field or isolated galaxies. As far as its physical meaning is concerned, the rest-frame $(U-B)$ color adopted in our analysis, bracketing the $4000 \AA$ break, can be used to study the average star formation histories over longer time-scales than emission lines indicators such as, e.g., [OII]. This choice could therefore provide clearer insights into mechanisms that operate on longer time scales, such as possibly those in action in dense environments as groups, where member galaxies have resided for a significant fraction of their lifetime.

Despite the apparent simplicity of this parameter, the origin of the physical mechanisms responsible for the variations in $F_{\text {blue }}$ in the group/cluster population still remains to be fully explained. In particular we are still unable to determine the relative influences of processes related to the environment and those that are intrinsic to the galaxy itself, and therefore the dichotomy between ab-initio/internal and external mechanisms responsible for the variation of $F_{\text {blue }}$ is still an open one. The fraction of galaxies on either sides of the bimodality in $(U-B)$ colors has been shown to depend strongly on galaxy luminosity and stellar mass (see, e.g., Baldry et al. 2004, 2006). Therefore, in studying the dependence of $F_{\text {blue }}$ on group environment, we define and adopt both luminosity volume-limited and mass volume limited samples.

In this section, we discuss the strategy adopted to correct for the $10 \mathrm{~K}$-sample incompleteness when measuring $F_{\text {blue }}$, the cutoff adopted in defining $F_{\text {blue }}$, and how we estimate errors on this quantity.

\subsection{Correcting for survey incompleteness}

The left panel of Fig. 1 shows that the coverage in Ra-Dec of the $10 \mathrm{~K}$-sample remains very uneven. While the mean sampling rate of the $10 \mathrm{~K}$-sample is around $\sim 30 \%$, this number varies significantly as a function of position: in the central regions the sampling rate is as high as $\sim 70 \%$, while it is as low as $\sim 10 \%$ in the regions near the borders. This unevenness can create problems when defining groups of homogeneous numerosity/richness irrespective of their position in the sky (see Sect. 6.1).

To correct for this problem we adopted for each galaxy a weighting scheme consisting of two factors: $\phi(m)$ and $\psi(\alpha, \delta)$. The first factor $\phi(m)$ is similar to one adopted for the luminosity and mass function estimates (see Zucca et al. 2009, for more details). It is obtained by a parabolic fit to the product $W$ of the inverse of the target sampling rate (TSR) and the inverse of the spectroscopic sampling rate $(S S R)$ :

$W=(1 / T S R) *(1 / S S R)$

$T S R$ is defined as $T S R=N_{\text {obs }} / N_{\text {phot }}$, the ratio of the total number of objects observed to the total number of potential targets, i.e., non-stellar objects in the parent bright photometric catalogue, (see Lilly et al. 2009). For the few compulsory targets observed in our survey (i.e., with forced slit positioning) $T S R$ was defined to equal 1. $S S R$ is defined as $\operatorname{SSR}(m)=N_{\text {spec }}(m) / N_{\text {obs }}(m)$ the ratio, calculated in bins of apparent magnitude, of the number of observed objects whose redshift was reliably measured to the total number of observed objects. The apparent magnitude dependence takes into account the progressive difficulty, moving toward fainter magnitudes, to measure a redshift. A more complex scheme, which includes the redshift dependence of SSR does not alter appreciably the final results (see Bolzonella et al. 2009).

The second factor $\psi(\alpha, \delta)$ corrects for the variation, as a function of $\mathrm{Ra}-\mathrm{Dec}$, of the mean correction factor expressed by $\phi(m)$. We estimated $\psi(\alpha, \delta)$ in two passes. In a grid of steps equal to $30^{\prime \prime}$ in right ascension and declination and in squares of $2^{\prime} \times 2^{\prime}$, we computed the ratio of the number of observed objects whose redshift was reliably measured to the total number of potential targets, as defined as above, within the same area. We then obtained $\psi(\alpha, \delta)$ by normalizing to unity the mean value of this ratio over the full $\mathrm{Ra}-\mathrm{Dec}$ coverage of the $10 \mathrm{~K}$-sample survey. The right panel of Fig. 1 shows in color-scale the resulting function $\psi(\alpha, \delta)$ before normalization. The parameters chosen in calculating this function allow us to reproduce well the inhomogeneities in the survey, even the vertical banding, visible in left panel of Fig. 1. To each galaxy we therefore assigned a weight: $w_{i}=\phi(m) \times \psi\left(\alpha_{i}, \delta_{i}\right)$ which is the galaxy weighting scheme used in the following analysis.

At the borders of the survey sampling is lower than average resulting both in higher galaxy weights and higher incompleteness in group detection. To alleviate this problem, we decided to restrict the analysis to the central area of the survey, where the inhomogeneity in sampling rate is significantly lower. This region is indicated by red lines in Fig. 1 and corresponds to galaxies within the following boundaries: $149.55 \leq \mathrm{Ra} \leq 150.42$, $1.75 \leq$ Dec $\leq 2.70$.

We note that our results are relatively insensitive to changes in the strategy used to define the weights, for example larger smoothing boxes in defining $\psi\left(\alpha_{i}, \delta_{i}\right)$. Even when no weights at all are used, our results are almost unchanged. A weighting scheme is needed when estimating in a homogeneous way group richness (for example when exploring trends of $F_{\text {blue }}$ as a function of groups richness). When dealing with the galaxy group population as a whole, the impact of the use of weights is minimal.

\subsection{Computing the blue fraction}

We divided galaxies into red and blue sub-samples taking advantage of the observed bimodality in galaxy $(U-B)$ restframe colors, visible in Fig. 4 (see also Cucciati et al. 2009). Accordingly, we defined blue galaxies as those with rest-frame colors $(U-B) \leq 1.0$. This value agrees with both the value chosen by Gerke et al. (2007) in their analysis of $F_{\text {blue }}$ in the DEEP2 groups sample and with the value adopted in a parallel analysis to our own by Cucciati et al. (2009b). We did not allow this value to vary with galaxy luminosity, as suggested for example by van Dokkum et al. (2000) and Blanton et al. (2006). Given the relatively small variation in $M_{B}$ of the bulk of our galaxy sample (of roughly $3 \mathrm{mag}$ ), the color-magnitude relationship quoted by these authors would imply a corresponding variation in the cut-off color value $\leq 0.1 \mathrm{mag}$, which we deemed to be negligible. 
A. Iovino et al.: The zCOSMOS redshift survey: how group environment alters global downsizing trends

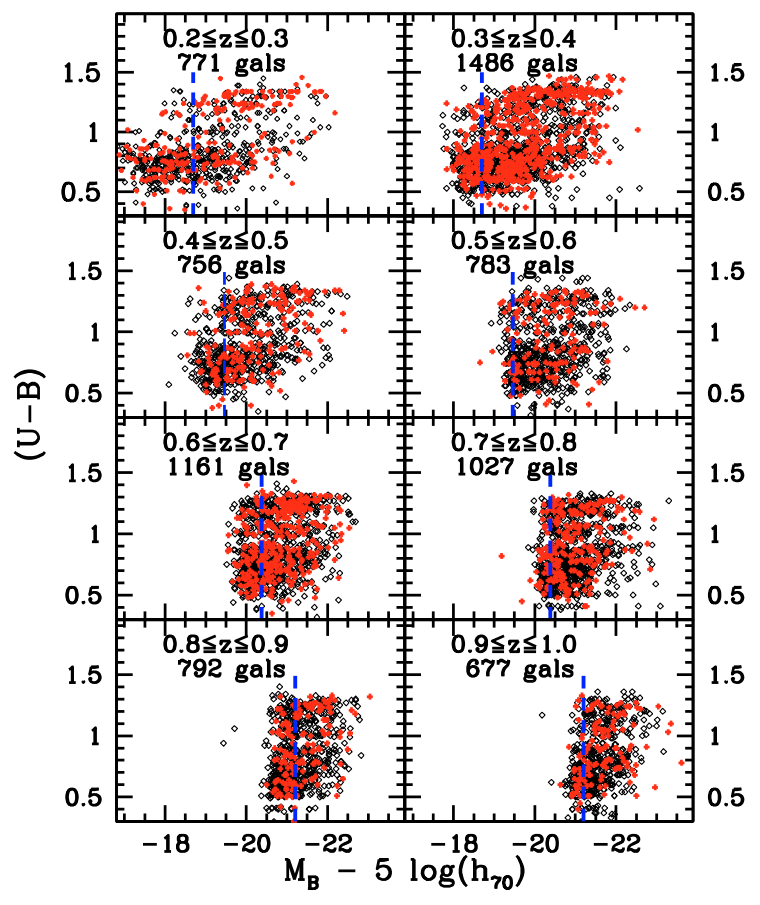

Fig. 4. Rest-frame $(U-B)$ colors plotted vs. $B$-band rest-frame magnitudes. In each panel redshift bins of width $\Delta z=0.1$ have been considered, as indicated by the labels, that list also the total number of galaxies located in each redshift bin. The points in red show galaxies located in groups according to the catalogue obtained from the full $10 \mathrm{~K}$-sample. The vertical dashed line in each panel indicates the absolute magnitude limits corresponding to the volume-limited sample that was chosen for galaxies contained in each redshift range.

From our data, there is no obvious evidence of evolution to redshift $\sim 1$ in the adopted cut-off value, and in our analysis we therefore decided to keep its value fixed with redshift.

After defining the cut-off value between red and blue galaxies we obtained a set of $N_{\mathrm{b}}$ blue galaxies from the total sample of $N_{\mathrm{t}}$ galaxies, each with a weight $w_{i}$. The corrected blue fraction was then given by:

$F_{\text {blue }}=\mathcal{N}_{\mathrm{b}} / \mathcal{N}_{\mathrm{t}}$

where the number of blue galaxies $\mathcal{N}_{\mathrm{b}}$ and the total number of galaxies $\mathcal{N}_{\mathrm{t}}$ are defined to be:

$\mathcal{N}_{\mathrm{b}}=\sum_{j=1}^{M} w_{j}, \quad \mathcal{N}_{\mathrm{t}}=\sum_{i=1}^{N} w_{i}$

where the index $j$ corresponds to all the blue galaxies, while the index $i$ corresponds to the full galaxy sample.

\subsection{Estimating errors in $F_{\text {blue }}$}

To estimate errors in the values computed for $F_{\text {blue }}$, we adopted a bootstrap re-sampling strategy. We randomly sampled by replacement the entire data set under consideration, e.g., all isolated galaxies in a given volume-limited sample. The error in $F_{\text {blue }}$ was then estimated to be the standard deviation in $F_{\text {blue }}$ distribution for 1000 such Montecarlo samples.

We used also the approximate analytical formulas provided by Gehrels (1986) to estimate the error in $F_{\text {blue }}$ but the differences in value with respect to the bootstrapping technique are minimal. In our plots we always show bootstrap errors.
Another source of errors and noise in our plots is cosmic variance. At lower redshifts, the volume sampled by zCOSMOS survey is not large enough to be considered a fair representation of the universal matter distribution. It is therefore possible that the presence of large scale structures introduces large fluctuations in the trends of $F_{\text {blue }}$ as a function of redshift, lowering significantly $F_{\text {blue }}$ at the redshift where these structures are located. Our survey shows quite a few of these prominent structures, for example those located at $z \sim 0.35$ and $z \sim 0.7$, readily visible in the top two panels of Fig. 2 (see also Kovac et al. 2009b). To alleviate this problem in our analysis we tried to adopt redshift bins large enough to smooth out as much as possible this effect.

\section{Defining luminosity volume-limited samples}

The zCOSMOS survey provides a unique data-set for measuring the evolution of the blue fraction up to $z \sim 1$. The excellent quality of the observed spectra prevent any possible bias against red, absorption lines only spectra (Lilly et al. 2009), while the simple $I_{A B} \leq 22.5 \mathrm{mag}$ limit used to select survey targets translates into a selection in the rest-frame $B$-band at $z \sim 0.8$. Therefore the zCOSMOS galaxy sample when rest-frame $B$-band selection is adopted is free from significant color-dependent incompleteness in $(U-B)$ rest-frame colors to the highest redshift bin explored.

However the reader should be warned that completeness in $B$-band rest frame selection does not imply completeness in, e.g., mass selection, as we will discuss at lenght in Sect. 7 and following. As a consequence any trend observed in restframe $B$-band selected samples needs to be re-examined when the selection criterion of the sample changes (see also, e.g., De Propris et al. 2004).

The absence of $(U-B)$ color incompleteness in zCOSMOS $B$-band volume limited samples can be visually appreciated in Fig. 4, where we plot for different redshift bins (as indicated in each panel) the rest-frame $(U-B)$ color versus rest-frame $B$-band absolute magnitude $M_{B}$.

In each panel distinctive red and blue populations of galaxies are visible, with loci that are separated approximately at $(U-B)=1$ at all redshifts. The cut-off in the galaxy population distribution visible on the left hand side of each panel is a consequence of zCOSMOS purely $I$-band flux-limited targetselection strategy and moves towards brighter magnitudes as the redshift increases. However, this progressively brighter cut-off does not introduce obvious biases against red galaxies as indicated by the cut-off line being nearly vertical in all panels with the possible exception of the last redshift bin, where the observed $I$-band begins moving blue-ward of the rest-frame $B$-band and a slight slanting of the cut-off in the galaxy population distribution starts becoming appreciable. Therefore, in this last redshift bin we need to be more conservative in the definition of the cut-off in absolute $B$-band rest-frame magnitude to avoid biases against red galaxies, even if this choice will result in smaller number of objects for our analysis.

Another factor to consider in our definition of volumelimited samples is that the typical galaxy luminosity evolves with redshift. We need to include an evolutionary term in our definition of cut-off magnitudes for the volume-limited samples because we aim to select a population of galaxies that is similar with respect to $M_{B}^{*}$ at all redshifts.

As suggested by the results obtained for the global luminosity function evolution of our sample (see Zucca et al. 2009, for 
Table 1. Summary of the four volume-limited data samples; $M_{\mathrm{ev}}^{*}=-20.3-5 \log h_{70}-1.1 z$.

\begin{tabular}{ccccc}
\hline \hline & Sample I & Sample II & Sample III & Sample IV \\
$M_{B}$ range & $M_{B} \leq M_{\mathrm{ev}}^{*}+2.1$ & $M_{B} \leq M_{\mathrm{ev}}^{*}+1.5$ & $M_{B} \leq M_{\mathrm{ev}}^{*}+0.8$ & $M_{B} \leq M_{\mathrm{ev}}^{*}+0.2$ \\
$z$ range & $0.1 \leq z \leq 0.45$ & $0.1 \leq z \leq 0.6$ & $0.1 \leq z \leq 0.8$ & $0.1 \leq z \leq 1.0$ \\
\hline All galaxies & 1798 & 2122 & 2616 & 2182 \\
Isolated galaxies & 315 & 442 & 431 & 326 \\
Group galaxies I & 676 & 670 & 709 & 447 \\
Group galaxies II & 218 & 237 & 412 & 447 \\
\hline
\end{tabular}

more details), the evolution in $M_{B}^{*}$ can be parametrized linearly by the equation

$M_{B \mathrm{ev}}^{*}=-20.3-5 \times \log \left(h_{70}\right)-1.1 \times z$

which includes an evolution with redshift of roughly 1 mag between $z \sim 0.1$ and $z \sim 1$ for $M_{B}^{*}$.

The galaxy luminosities quoted from now on are always evolutionary-corrected present-day luminosities to ensure that galaxies of similar luminosity are being compared in different redshifts bins.

We defined four different luminosity volume-limited samples, from sample I to sample IV, each covering progressively higher ranges of redshift, and defined by evolving the cut-off magnitudes $M_{\text {cut-off }}=M_{B \mathrm{ev}}^{*}+2.1 /+1.5 /+0.8 /+0.2$, as illustrated in Fig. 5.

Table 1 summarizes the properties of these four different volume-limited samples: the different redshift ranges covered and the total numbers of galaxies and isolated/group galaxies contained within the Ra - Dec limits described in Sect. 3.1.

From now onwards when we will talk of the field population we always mean the total galaxy sample, i.e., the full galaxy population including group/isolated galaxies.

We note that while the full group catalogue was obtained using the entire 10K-sample galaxy catalogue, for each of the volume-limited samples defined above we selected a corresponding uniform sample of groups possessing at least two member galaxies brighter than the $B$-band rest-frame $M_{\text {cut-off }}$ considered (Group galaxies I). This strategy avoids the redshift inhomogeneity introduced in our group catalogue by the progressive brightening of the rest-frame $B$-band magnitudes sampled by the survey as redshift increases. A given group will have a different number of members in each volume-limited sample, but within each volume-limited sample group's numerosity/richness will be measured consistently at all redshifts. Unless explicitly mentioned when we talk of group galaxies, we refer to Group galaxies I.

We also introduced a further set of galaxy groups: those that possess at least two members in sample IV (Group galaxies II). By studying the variation in $F_{\text {blue }}$ for galaxies of different luminosities that are members of this group sample one can hope to disentangle the effect of galaxy luminosity on $F_{\text {blue }}$ from that of group richness: this is because the groups in this sample should be homogeneous in terms of richness as a function of redshift, irrespective of the magnitude of the member galaxies considered in the analysis (see Sect. 5). For the sake of robustness, the value of the group observed line-of-sight velocity dispersion $\sigma$, whenever used in our analysis, is always estimated using all observed group members, irrespective of their absolute magnitude.

After defining galaxy weights, volume-limited samples and the corresponding group/isolated subsets, we proceeded to estimate $F_{\text {blue }}$, the fraction of blue galaxies, for each galaxy sample and its dependence on group properties, galaxy luminosity, and redshift.

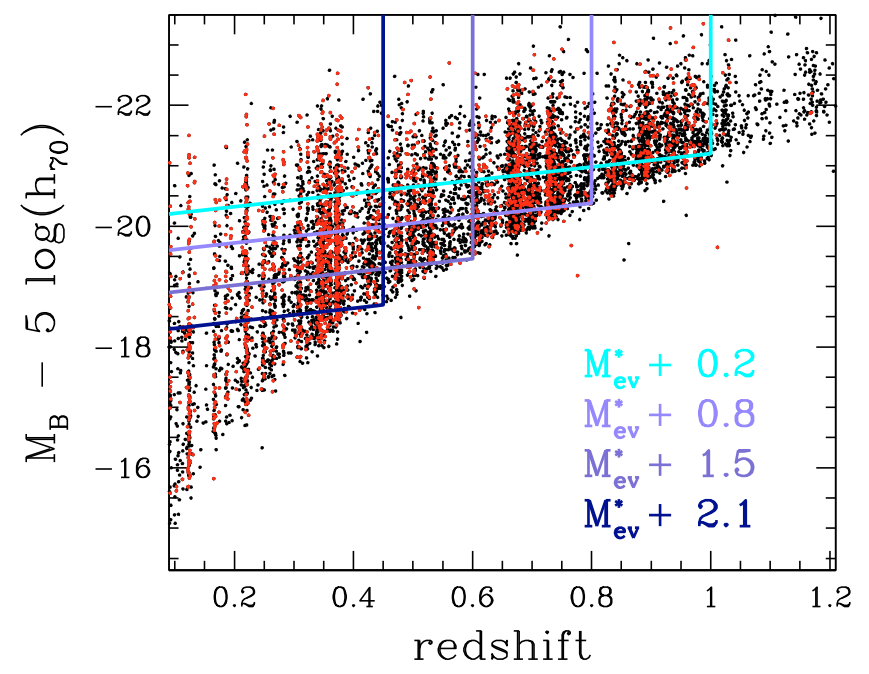

Fig. 5. Redshift distribution of the 10K-sample zCOSMOS galaxies. Red points represent galaxies located in groups. The lines drawn correspond to the four different volume-limited samples discussed in the text. We assumed $M_{\mathrm{ev}}^{*}=-20.3-5 \times \log \left(h_{70}\right)-1.1 \times z$ and the different labels and the lines drawn correspond to the four different volume-limited samples discussed in the text.

\section{Blue fraction as a function of galaxy luminosity and environment up to $z \sim 1$}

In the local Universe, the correlation between galaxy luminosities and colors is a well-known observational result: more luminous galaxies have typically redder colors than less luminous galaxies (see Baldry et al. 2004, and references therein). A similar color segregation has been observed between local groups and field samples: redder galaxies are preferentially located in galaxy groups and clusters (see De Propris et al. 2004, and references therein). It is therefore interesting to use our sample to check whether these trends survive at higher redshifts and if they show weakening or even any visible reversal.

A similar analysis was performed using DEEP2 data for the redshift range $0.75 \leq z \leq 1.3$ by Gerke et al. (2007), and using VVDS data for the range $0.25 \leq z \leq 1.2$ by Cucciati et al. (2009b). The VVDS and DEEP2 surveys were the first to use in their investigation a homogeneous dataset from the lowest to the highest redshift bins explored, and a group sample spanning a wide range of richnesses, down to poorest systems, in contrast to previous work that mainly considered higher richness, and more easily detectable, systems. With respect to these two pioneering large high-redshift surveys, zCOSMOS presents some non-negligible advantages. We have a larger volume coverage enabling us to complete more robust statistical analyses than VVDS, and smaller errors in galaxy redshift measurements - around $275 \mathrm{~km} \mathrm{~s}^{-1}$ for VVDS, (see Le Fèvre et al. 2005), which allows us to compile a group catalogue that is less prone 


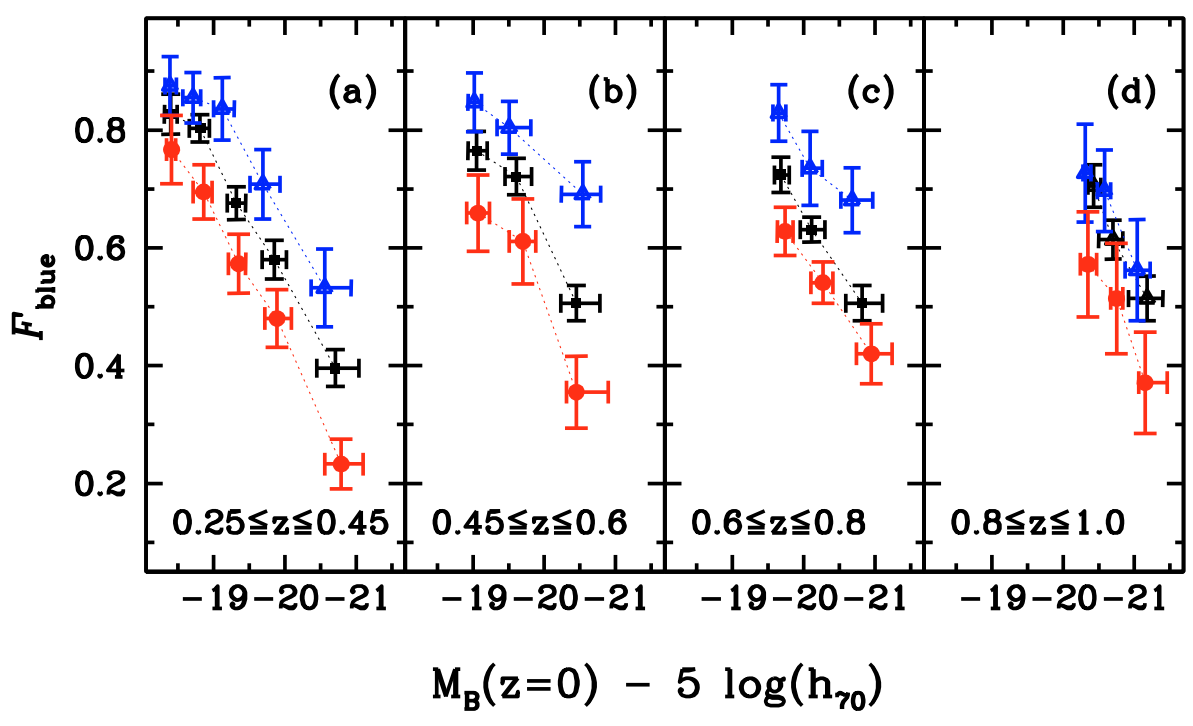

Fig. 6. The four panels show $F_{\text {blue }}$ in different redshift bins, as indicated on the bottom of each panel, as a function of absolute luminosity, evolution corrected to $z \sim 0$ to ensure that similar galaxies are being compared across different redshifts bins. Different colors refer to different galaxy samples: red circles refer to group galaxies, blue triangles to isolated galaxies, while black squares to the total galaxy population. The errors on $F_{\text {blue }}$ are obtained using bootstrapping, while the error bars along the luminosity axis link the upper and lower quartiles of the luminosity distribution of galaxies within the bin considered. At all magnitudes and at all redshifts groups contain less blue galaxies than the field and the isolated galaxies population. For all redshift bins considered and irrespective of the environment fainter galaxies are always bluer than brighter galaxies. For all environments $F_{\text {blue }}$ increases with redshift. to contamination and incompleteness, especially for low richness and low velocity dispersion systems. We are also less plagued by the color incompleteness (and, more importantly for the subsequent analysis, mass incompleteness) that affects DEEP2 data in the redshift range covered by their analysis, and have the ability to cover the complete redshift range $0.2 \leq$ $z \leq 1.0$, monitoring the redshift evolution in $F_{\text {blue }}$ in a continuous way.

As a first step, we explored how $F_{\text {blue }}$ varies with galaxy luminosity. We defined four independent redshift bins as shown in Fig. 6: [0.25:0.45], [0.45:0.6], [0.6:0.8], [0.8:1.0]. Within each of these redshift intervals and using the volume-limited samples defined in Table 1, we defined sub-samples of galaxies in independent bins of galaxy luminosities. The binning in galaxy luminosity was chosen in such a way to ensure a sizeable number of galaxies in each environment and redshift bin considered. $F_{\text {blue }}$ and its error bar were estimated using the procedures described in Sect. 3, while the error bars drawn along the luminosity axis link the upper and lower quartiles of the luminosity distribution of galaxies within each bin.

Figure 6 shows the results obtained for the different galaxy samples: red circles for group galaxies, blue triangles for isolated galaxies, and black squares for the total galaxy population. In each redshift bin all the different galaxy populations display a decrease in the fraction of blue galaxies for increasing restframe galaxy luminosities, while at a fixed luminosity bin, blue galaxies are always less common in the group environment than in the field and most common among the isolated galaxy population. Figure 6 therefore suggests that at all redshifts explored the color of galaxies at a given luminosity becomes redder earlier in groups than in the field or in lower density regions.

Furthermore, the differences between the galaxy population of the three different environments seem to increase at higher luminosities in each of the four panels of Fig. 6 and this result echoes a similar one in Cucciati et al. (2006).

Towards redshift $z \sim 1$ the differences among the three environments progressively decrease. However up to the highest redshift bin explored we do not see any hint of a possible reversal of the trend of $F_{\text {blue }}$ as a function of luminosity, a robust result as our sample is free from significant color-dependent incompleteness up to $z \sim 1$ (see Sect. 4). Such possible trend reversal was tentatively detected by Gerke et al. (2007), albeit with large error bars, for the redshift bin $0.7 \leq z \leq 1.0$ and for magnitudes brighter than $M_{B} \sim-21.5$.
We used the four volume-limited samples and the three galaxy samples defined in Table 1 to explore in better detail the redshift trends implied by Fig. 6. For each of these samples, we plotted $F_{\text {blue }}$ as a function of redshift in Fig. 7, to help determine directly whether the rate of variation in $F_{\text {blue }}$ differs significantly in groups compared to the field/isolated galaxy population. Each panel refers to a volume-limited sample defined by the labels at its bottom, where red circles indicate $F_{\text {blue }}$ for group galaxies, while black squares and blue triangles show the same quantity for field and isolated galaxies, respectively.

The first piece of information conveyed by Fig. 7 is that color segregation appears to be already in place at $z \sim 1$ : panel (d) shows that even in the highest redshift bin explored there is a small, but significant, difference in $F_{\text {blue }}$ among the different galaxy samples, mirroring the information provided by panel (d) of Fig. 6. Furthermore for each of the luminosity bins explored color segregation increases with cosmic time, as the differences of $F_{\text {blue }}$ in the group, field and isolated galaxy populations increase significantly moving from high to low redshifts.

These results are in good agreement with those from the VVDS survey presented by Cucciati et al. (2009b). However we seem to detect evolution in $F_{\text {blue }}$ across the range $0.75 \leq$ $z \leq 1.0$, in contrast with the results of Gerke et al. (2007) using DEEP2 data-set. We note that comparing directly our panel (d) of Fig. 7 with the first panel of their Fig. 7, where the magnitude ranges explored are quite similar and the sample analyzed is purely volume-limited as in our analysis, the disagreement is not so evident.

We chose to parametrize the evolution in $F_{\text {blue }}$ with redshift with a law of the form $F_{\text {blue }} \propto(1+z)^{\beta}$. The results of the best fit solutions obtained with this parametrization are given in Table 2 and shown as dashed lines in Fig. 7. These lines tend to diverge between high and low $z$. Toward higher redshift, one can consider whether, irrespective of the environment considered, most galaxies in the luminosity ranges explored resided in the blue cloud, while the red sequence remained more or less empty. As cosmic time increases, the blue cloud may then become progressively depleted and the rate at which this depletion occurs seems to be higher in higher density environments, implying that the star-formation rate is declining more rapidly in groups and clusters.

While the extrapolated values of $F_{\text {blue }}$ at $z \sim 0$ vary as a function both of environment and the luminosity cut-off considered, the values of $\beta$ do not exhibit any appreciable differences 


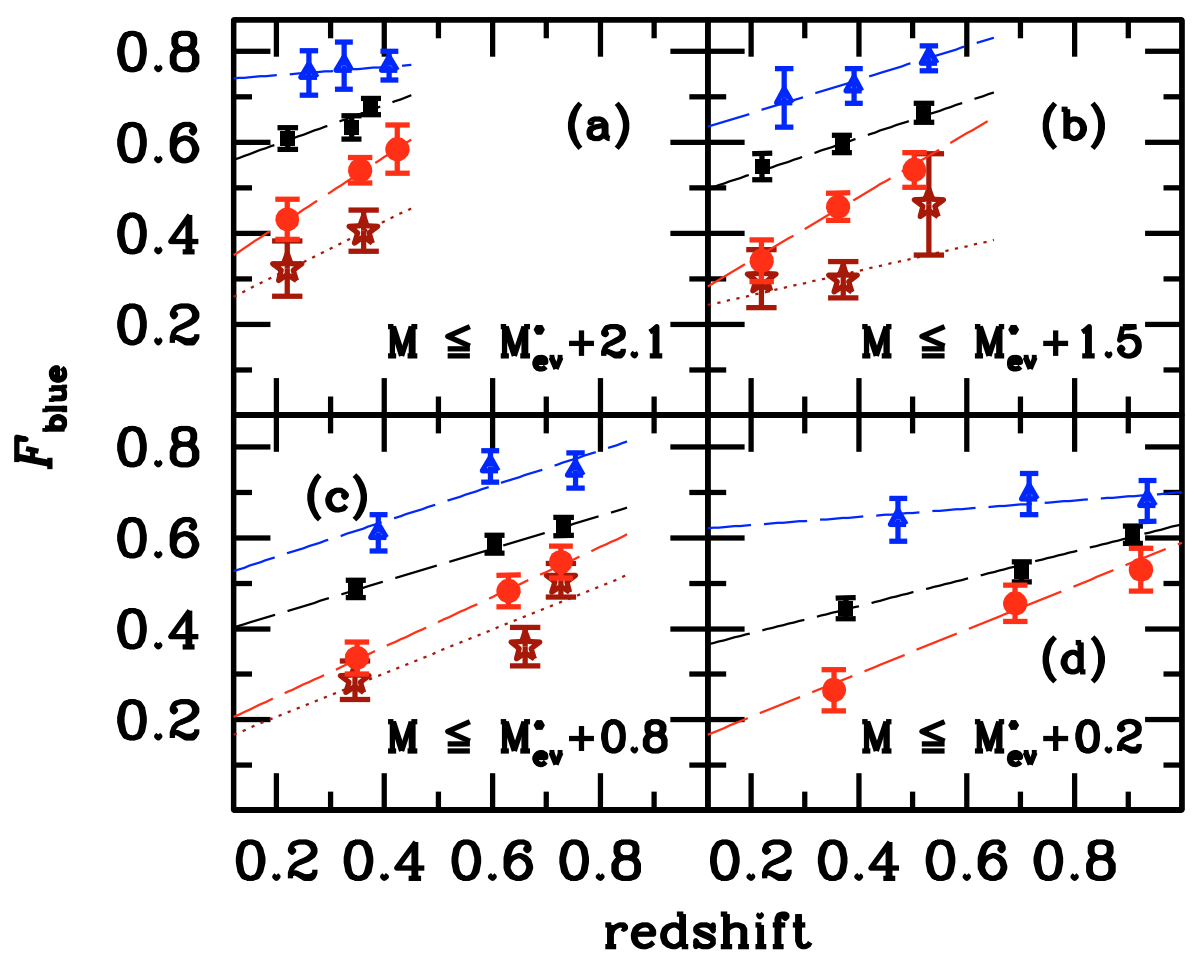

Fig. 7. The four panels show $F_{\text {blue }}$ as a function of redshift for each of the different volumelimited samples defined in Table 1. The label in each panel indicates the range in evolution corrected, present-day, absolute magnitude for the galaxies plotted (we assumed $M_{\mathrm{ev}}^{*}=-20.3-$ $5 \log h_{70}-1.1 z$ ). Red circles refer to group galaxies, blue triangles to isolated galaxies, while black squares to the total galaxy population. Brown stars are those corresponding, for each volume-limited sample, to the population of galaxies in groups with at least two members in Sample IV, that is sample Group galaxies II in Table 1. Color segregation is already in place at redshift $\sim 1$ and increases sensibly moving from higher to lower redshifts for all the volume limited samples considered. See text for more details.

Table 2. Summary of fits results for $F_{\text {blue }}$ as a function of redshift in the volume-limited samples defined in Table $1 ; M_{\mathrm{Ev}}^{*}=-20.3-5 \times \log \left(h_{70}\right)-$ $1.1 \times z$.

\begin{tabular}{|c|c|c|c|c|c|c|c|c|}
\hline \multirow[t]{2}{*}{$\begin{array}{c}M_{B} \text { range } \\
z \text { range } \\
\end{array}$} & \multicolumn{2}{|c|}{$\begin{array}{c}\text { Sample I } \\
M_{B} \leq M_{B \mathrm{Ev}}^{*}+2.1 \\
0.1 \leq z \leq 0.45\end{array}$} & \multicolumn{2}{|c|}{$\begin{array}{c}\text { Sample II } \\
M_{B} \leq M_{B \mathrm{Ev}}^{*}+1.5 \\
0.1 \leq z \leq 0.6\end{array}$} & \multicolumn{2}{|c|}{$\begin{array}{c}\text { Sample III } \\
M_{B} \leq M_{B \mathrm{Ev}}^{*}+0.8 \\
0.1 \leq z \leq 0.8\end{array}$} & \multicolumn{2}{|c|}{$\begin{array}{c}\text { Sample IV } \\
M_{B} \leq M_{B \mathrm{Ev}}^{*}+0.2 \\
0.1 \leq z \leq 1.0\end{array}$} \\
\hline & $F_{\text {blue }}(z=0)$ & $\beta$ & $F_{\text {blue }}(z=0)$ & $\beta$ & $F_{\text {blue }}(z=0)$ & $\beta$ & $F_{\text {blue }}(z=0)$ & $\beta$ \\
\hline All galaxies & $0.51 \pm 0.06$ & $0.88 \pm 0.40$ & $0.45 \pm 0.04$ & $0.92 \pm 0.27$ & $0.36 \pm 0.03$ & $0.99 \pm 0.20$ & $0.32 \pm 0.03$ & $0.96 \pm 0.18$ \\
\hline Isolated galaxies & $0.73 \pm 0.15$ & $0.16 \pm 0.67$ & $0.58 \pm 0.10$ & $0.69 \pm 0.44$ & $0.48 \pm 0.08$ & $0.85 \pm 0.36$ & $0.60 \pm 0.12$ & $0.23 \pm 0.36$ \\
\hline Group galaxies I & $0.29 \pm 0.08$ & $2.01 \pm 0.87$ & $0.24 \pm 0.06$ & $2.05 \pm 0.67$ & $0.19 \pm 0.05$ & $1.97 \pm 0.50$ & $0.16 \pm 0.05$ & $1.87 \pm 0.53$ \\
\hline Group galaxies II & $0.21 \pm 0.12$ & $2.09 \pm 1.85$ & $0.19 \pm 0.08$ & $1.75 \pm 1.27$ & $0.14 \pm 0.05$ & $2.20 \pm 0.61$ & $0.16 \pm 0.05$ & $1.87 \pm 0.53$ \\
\hline
\end{tabular}

between the different environments as a function of the chosen luminosity cut-off. On the other hand, there is a noticeable increase in the value of $\beta$ moving from isolated to group galaxies, although the error bars are quite large.

Since $\beta$ implies that there is a fractional decrease in $F_{\text {blue }}$ with cosmic time (or, alternatively, a fractional increase of the percentage of red galaxies with cosmic time) this result suggests that we detect the signature of an environmental dependence of the variations in $F_{\text {blue }}$ with cosmic time. However the mechanisms responsible for the environmental trends that we witness cannot be accurately constrained.

Our results could be the consequence of physical mechanisms operating in the denser group environment or simply the result of an ab initio bias relating galaxy luminosity/mass and its environment. In other words, we could be witnessing the more rapid quenching of star formation - and, as a consequence, the faster build-up of the red sequence - in denser environments, or the delayed and more efficient replenishing of the blue cloud in lower density environments. We will return to this point in the following sections.

The group points in Fig. 7 do not correspond to a group population that is homogeneous across each of the four different panels but to the samples indicated as Group galaxies I in Table 1, i.e., galaxies in groups of more than two members observed within the volume-limited sample plotted in each panel. Moving from panel (a) to panel (d) in Fig. 7, we consider groups that are intrinsically more rich, since they possess two or more members at progressively brighter cut-off magnitudes. Accordingly the observed decrease in $F_{\text {blue }}$ for the group galaxy population between the first and the last panel of Fig. 7 is the result of two different effects: the brightening of the galaxy population, an effect easily visible also for the isolated and the field samples, and the increasing richness of groups observed in the brighter volume-limited samples.

It is therefore interesting to remove the richness-dependent effect from this plot and to compare galaxies residing in groups of homogeneous richness across the different volume-limited samples, using the sample of groups with two or more members brighter than $M_{B} \leq M_{B \mathrm{Ev}}^{*}+0.2$, i.e., the brightest absolute magnitude cut-off in our sample. This way, we select for each panel, except panel (d) whose group sample remains unchanged by definition, a set of groups richer than those considered before. Obviously, once a group survives in the catalogue defined by the more stringent luminosity cut-off, we then plot in the appropriate panel all its members observed within the volume-limited sample under study. The result of this exercise is shown by the brown stars in Fig. 7 and by the last row of Table 2, labeled Group galaxies II. In this case, the groups considered are homogeneous in richness up to $z \sim 1$. The difference compared to the field population (and the isolated galaxies) increases significantly for 
these richer groups. In contrast the dependence of $F_{\text {blue }}$ on the rest-frame $B$ magnitude of the group population is reduced significantly. Table 2 shows that the slopes of the fits to the Group galaxies II points are virtually indistinguishable among themselves, irrespective of the luminosity limits adopted in each of the four different panels.

\section{Blue fraction as a function of group properties to $z \sim 1$}

The results obtained in the previous section using Group galaxies II samples suggest that group richness is an important ingredient in setting the value of $F_{\text {blue }}$, possibly more influential than the galaxy rest-frame $B$ magnitude.

Group richness can be considered, albeit with a large scatter, a proxy for the mass of the halo where the group resides (see Knobel et al. 2009). Therefore, the results just obtained echo at higher redshifts findings in the local Universe by Weinmann et al. (2000). Using both galaxy colors and specific star formation rate indicators to define samples of early/late type galaxies, these authors showed that at fixed halo mass the dependence of the galaxy type fraction on luminosity is quite weak.

Concerning the dependence of $F_{\text {blue }}$ on group richness and/or velocity dispersion - another possible proxy of halo mass many conflicting observational results, however, exist in the literature. Some authors have claimed the presence of a relationship between group and galaxy properties (see, e.g., Biviano et al. 1997; Zabludoff \& Mulchaey 1998; Margoniner et al. 2001; Martínez et al. 2002; Goto et al. 2003; Tanaka et al. 2004; Poggianti et al. 2006; De Lucia et al. 2007; Gerke et al. 2007; Koyama et al. 2007, to quote a few), while other authors have claimed that such relationships are not present (see, e.g., Smail et al. 1998; Ellingson et al. 2001; Fairley et al. 2002; De Propris et al. 2004; Goto 2005; Wilman et al. 2005b; Popesso et al. 2007).

In the following, we explore with our sample the dependence of $F_{\text {blue }}$ on group richness and velocity dispersion.

\subsection{Blue fraction as a function of group richness}

The use of the term "richness" for galaxy clusters dates back to Abell, who introduced a broad classification of clusters in three richness classes based on counting galaxies between $m 3$ and $m 3+2 \mathrm{mag}$, where $m 3$ is the magnitude of the thirdbrightest galaxy (see Abell 1958). In this paper, we use the term richness for each group to simply indicate the number of members observed in each of the different volume-limited samples. As such, the richness of a group is not an absolute number, but varies depending on the absolute magnitude cut-off chosen to define the sample of groups. A possible better name for this quantity could be group numerosity. In estimating group richness/numerosity, however, even within this more limited definition, one has to consider corrections to the number of galaxy members observed, to properly account for the large-scale variations in the mean sampling rate of the $10 \mathrm{~K}$-sample.

While the mean sampling rate of the $10 \mathrm{~K}$-sample is about $\sim 30 \%$ and increases up to $\sim 40 \%$ in the restricted central area adopted for our analysis, there are large spatial variations in this number (see Fig. 1).

To correct for this problem, we estimated richness using the weighting scheme discussed in Sect. 3.1. For each volume limited sample we simply added galaxy weights to count the group

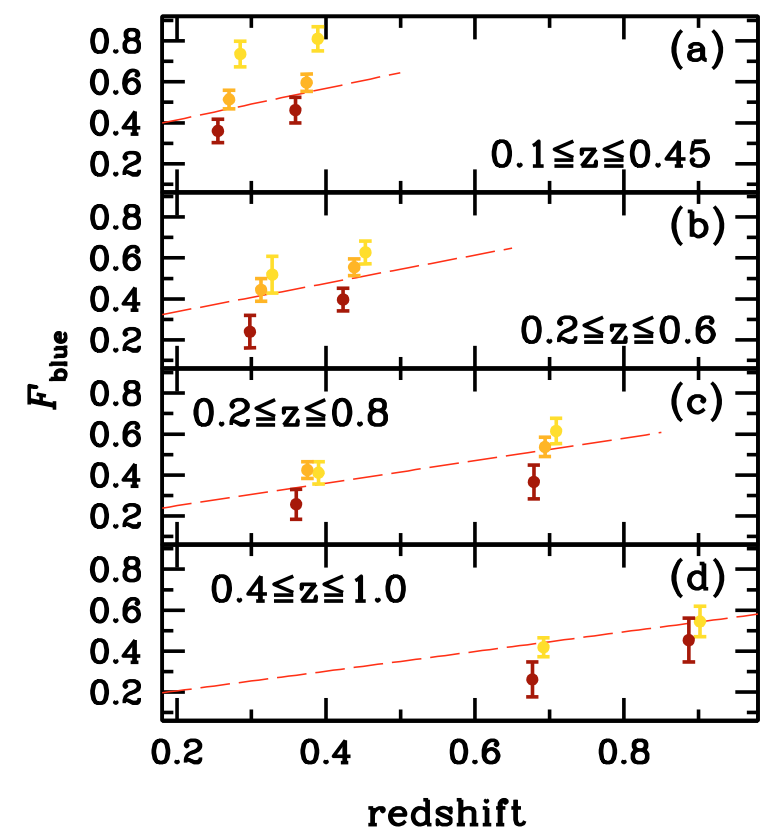

Fig. 8. Dependence of the blue fraction $F_{\text {blue }}$ on group redshift and richness. In each panel, the different redshift ranges indicated by the label are considered. In panels a)-c), we plot the value of $F_{\text {blue }}$ for groups with $N_{\text {corr }} \leq 4$, yellow points, $4<N_{\text {corr }} \leq 10$, orange points and $N_{\text {corr }}>10$, brown points, in samples I-III respectively. In panel d) we plot the value of $F_{\text {blue }}$ for groups in sample IV with $N_{\text {corr }} \leq 8$, yellow points and $N_{\text {corr }}>8$ brown points. The points have been slightly offset in redshift for the sake of clarity. In all panels, the red dashed line corresponds to fits obtained for the entire galaxy group population, as shown in Fig. 7. There is a consistent trend for all the samples and all the redshift bin explored: groups with higher $N_{\text {corr }}$ tend to have a lower fraction of blue galaxies, a trend superimposed on the global decrease of $F_{\text {blue }}$ moving from high to low redshift.

members, by writing richness as $\mathcal{N}_{\text {corr }}=\sum_{j=1}^{M} w_{j}$, where $M$ is the number of members observed in each volume-limited sample.

Figure 8 shows the dependence of the value of $F_{\text {blue }}$ on group richness. In each panel, we consider different redshift ranges, corresponding to the four volume-limited samples defined in Table 1, and divide the corresponding group sample according to richness. In all panels, the red dashed line indicates the fit to the global galaxy population obtained in Sect. 5. In the first three panels, the yellow points correspond to groups of observed richness $\mathcal{N}_{\text {corr }} \leq 4$, the orange points to groups of observed richness $4<\mathcal{N}_{\text {corr }} \leq 10$ and the brown points to groups of observed richness $\mathcal{N}_{\text {corr }}>10$. In panel (d), yellow points correspond to groups of observed richness $\mathcal{N}_{\text {corr }} \leq 8$ and brown points to groups of observed richness $\mathcal{N}_{\text {corr }}>8$. The limits chosen to divide the groups into bins of richness are arbitrary but ensure that each richness bin contains a sizeable number of group member galaxies (always above $\sim 30$ ).

The main result presented in Fig. 8 is that for all redshifts explored and volume limited samples, $F_{\text {blue }}$ decreases monotonically between higher and lower redshifts and richer groups have a lower value of $F_{\text {blue }}$. This result is in agreement with similar trends observed in the local Universe (see Margoniner et al. 2001; Goto et al. 2003).

We noted in Sect. 2.2 that in our sample we do not have any more massive relaxed clusters, especially at low redshift, but instead we probe mainly the poorer clusters and group environment. For our lower richness groups, the results obtained - especially in the lowest redshift bin, where we are dealing with the poorest groups of the sample in absolute terms - blend with 
those observed for the global field population. We may be concerned that these results are just the by-product of a higher interloper fraction for these (poorer) groups. However, as discussed in detail in Knobel et al. (2009), the interloper fraction of our group catalogue shows a minimal increase when the number of detected members decreases. Furthermore in the local Universe extremely poor groups are known to be dominated by spiral galaxies (Zabludoff \& Mulchaey 1998), and so what we are witnessing is most probably a real physical trend.

\subsection{Blue fraction as a function of group velocity dispersion}

We binned our group sample using also the observed line-ofsight velocity dispersion $\sigma$, another possible proxy for group mass. The estimate of $\sigma$ is difficult, especially when one is dealing, as in our case, with groups of only a few members with measured redshifts (see Beers et al. 1990). However, restricting the analysis to groups with observed numbers of members equal or greater than 5 allows us to observe a reasonable correlation between $\sigma$ and halo group mass (Knobel et al. 2009). We therefore used only groups with at least 5 members with measured redshifts in the $10 \mathrm{~K}$-sample to explore how $F_{\text {blue }}$ depends on the measured $\sigma$ in different redshift bins and for different volumelimited samples.

Our results are shown in Fig. 9. In panel (a), the yellow point indicates $F_{\text {blue }}$ for groups with $\sigma \leq 250 \mathrm{~km} \mathrm{~s}^{-1}$, the orange point for groups with $250 \leq \sigma \leq 550 \mathrm{~km} \mathrm{~s}^{-1}$, and the brown point for groups with $\sigma>550 \mathrm{~km} \mathrm{~s}^{-1}$. In the three remaining panels, the yellow points show $F_{\text {blue }}$ for groups with $\sigma \leq 350 \mathrm{~km} \mathrm{~s}^{-1}$, the orange points for groups with $350 \leq \sigma \leq 650 \mathrm{~km} \mathrm{~s}^{-1}$, and the brown points for groups with $\sigma>650 \mathrm{~km} \mathrm{~s}^{-1}$. In all panels, the red dashed line corresponds to the fits obtained for the entire galaxy groups population in the volume-limited sample, as in Fig. 8, while the red points are those corresponding to the sample of groups detected with at least 5 members in the flux-limited sample irrespective of their velocity dispersion. Figure 9 shows that there is a consistent trend in all panels: groups with higher velocity dispersion tend to have a lower fraction of blue galaxies.

Limiting the sample in the analysis only to groups of at least 5 observed members, produces the systematic offset towards lower $F_{\text {blue }}$ observed in each of the panels when comparing the red points to the dashed red lines: with the requirement of $N \geq 5$ we remove from the sample the poorer groups in each volumelimited sample.

We conclude this section with a word of caution. One would be tempted to compare points as a function of redshift across the last three different panels, since the $\sigma$ cut-off chosen is equal for each of them, and after showing in Sect. 8.2 that galaxy luminosity is of much less importance in determining $F_{\text {blue }}$ than group richness. This comparison would apparently result in a statement of non-evolution of $F_{\text {blue }}$ as a function of redshift for groups of similar velocity dispersion.

However one needs to be extremely careful when comparing results obtained at different redshifts. As cosmic time increases a system will experience an increase in its velocity dispersion, as its halo mass becomes higher due to structure growth (see, e.g., the prescriptions obtained by using semi-analytic models by Wechsler et al. 2002 and Poggianti et al. 2006, for an application). Furthermore, one should keep in mind that the imposed cut-off on the number of members observed in the fluxlimited sample is expected to introduce a strong bias favoring richer groups moving from panel (a) to panel (d), as the absolute luminosity of the observed galaxies becomes brighter. What appears as an absence in evolution of $F_{\text {blue }}$ as a function of

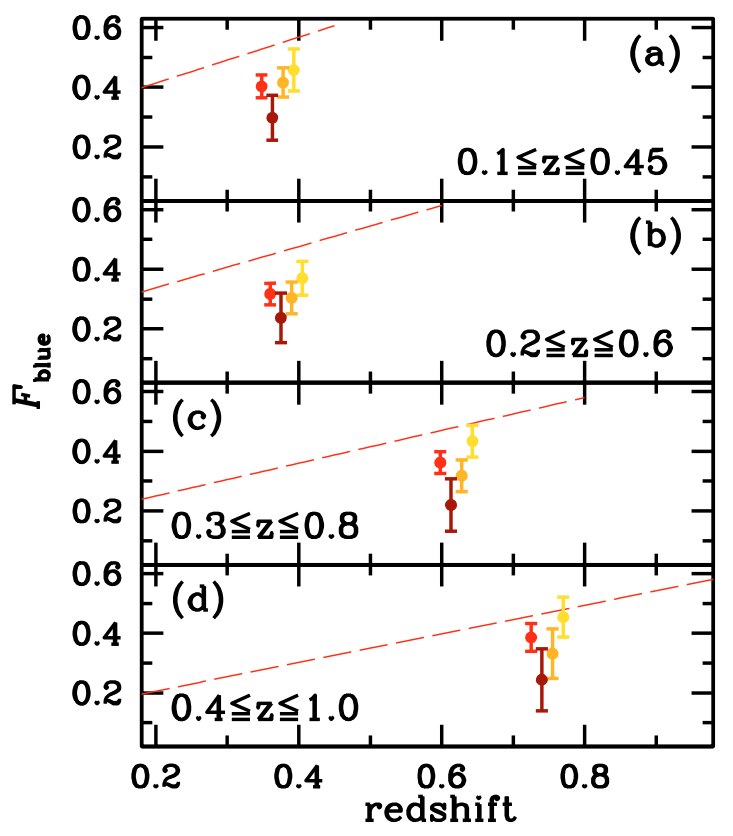

Fig. 9. Dependence of the blue fraction $F_{\text {blue }}$ on redshift and line-ofsight velocity dispersion of groups. In each panel, the different redshift ranges indicated by the label are considered. In all panels only groups detected with at least 5 members in the flux-limited sample used by the group detection algorithm are plotted, to avoid the large uncertainty in velocity dispersion measurement when poorer structures are considered. In panel a), the yellow point shows $F_{\text {blue }}$ for groups with $\sigma \leq$ $250 \mathrm{~km} \mathrm{~s}^{-1}$, the orange point for groups with $250 \leq \sigma \leq 550 \mathrm{~km} \mathrm{~s}^{-1}$, and the brown point for groups with $\sigma>550 \mathrm{~km} \mathrm{~s}^{-1}$. In the three remaining panels, the yellow points show $F_{\text {blue }}$ for groups with $\sigma \leq 350 \mathrm{~km} \mathrm{~s}^{-1}$, the orange points for groups with $350 \leq \sigma \leq 650 \mathrm{~km} \mathrm{~s}^{-1}$, and the browns point for groups with $\sigma>650 \mathrm{~km} \mathrm{~s}^{-1}$. In all panels, the red dashed line corresponds to fits obtained for the entire galaxy group population in the volume-limited sample, as in Fig. 8, while the red points are those corresponding to the sample of groups detected with at least 5 members in the flux-limited sample, irrespective of their measured line-of-sight velocity dispersion. In each plot, the points are located in redshift at the median value of the sample considered, with a small offset, for the sake of clarity, among the different $\sigma$ limited samples. There is a consistent trend for all the redshift bins explored: groups with higher line-of-sight velocity dispersion tend to have a lower fraction of blue galaxies.

redshift at a fixed velocity dispersion is thus at least partly caused by the progressive bias against lower richness groups moving from panel (a) to panel (d).

Estimates of group richness and group velocity dispersion often have large error bars, due to the paucity of member-galaxy samples available, and group richness/group velocity dispersion are properties that have a large scatter in their relationship to more fundamental quantities - as the mass of the halo where the group resides. As a consequence, it is unsurprising to observe a large scatter in the trends that relate group richness and group velocity dispersion with the value of $F_{\text {blue }}$.

To avoid producing biased results on evolution proper care has to be taken to compare properties of group samples that are truly homogeneous in the different redshift bins explored.

\section{Moving from luminosity to stellar mass: redefining the Butcher-Oemler effect}

The results obtained in the previous section can all be interpreted in the framework of the classical Butcher-Oemler effect (Butcher \& Oemler 1978), in its wider context extended to group population (see Allington-Smith et al. 1993). 
Taking advantage of the wide redshift and galaxy/group population coverage of the $10 \mathrm{~K}$-sample galaxy/groups catalogue, we have been able to show that the blueing of the galaxy population in groups/clusters when moving to higher redshift, originally observed by these authors thirty years ago, is a real effect, which differs from that observed for the global galaxy population. It also exhibits specific trends as a function of both galaxy $B$-band rest-frame luminosity and group properties. These trends are present as a function of environment in all luminosity and redshift bins explored and seem to become progressively more conspicuous moving from $z \sim 1$ to lower redshifts and from lower to higher luminosities.

However almost galaxy properties depend strongly on galaxy stellar mass, and this is true particularly for galaxy colors. Galaxy stellar mass in turn is known to correlate with environment and can be a key player in determining galaxy properties and in linking them to the environment in which they reside.

It is therefore important to check whether the strong effects evident in luminosity-selected samples are still present when the analysis is repeated using stellar-mass-selected samples. In this way, we probe the possibility of these effects being the distorted/amplified reflection - related to the biased view imposed by the luminosity selection - of more fundamental relationships either between masses and environment, or between masses and galaxy colors.

In the following sections, we re-examine the original Butcher-Oemler results using mass-limited samples instead of volume-limited samples. What becomes of the observed strong trends in $F_{\text {blue }}$ as a function of environment and redshift, shown in, e.g., Fig. 7, when one utilizes samples complete in mass? Are we able to confirm the existence of a higher proportion of blue galaxies in higher redshift groups with respect to their lower redshift counterparts, when using mass-limited samples? Can we still see an excess of red galaxies in groups with respect to the field/isolated galaxy population even when analyzing mass bins?

Obviously analyzing volume-limited stellar-mass selected samples involves a significant reduction in the galaxy sample size available to our study, as the selection of mass-complete samples implies the rejection of a large number of low-mass galaxies for which our $I_{A B}$ selected redshift survey is incomplete. However this is an unavoidable step in clarifying the key mechanisms determining the relationships observed for luminosityselected samples.

Galaxy stellar mass has the further advantage of being more stable than its luminosity. The $B$-band galaxy rest-frame luminosity may indeed change dramatically during a galaxy lifetime because of bursts of star-formation. Even in the absence of these bursts, the rest-frame $B$-band luminosity evolves with redshift, possibly in different ways for different galaxy populations, and one needs to introduce - as we have - an average evolution correction term to sample homogeneous galaxy populations in the different redshift bins explored. On the other hand, stellar mass varies to a far lesser extent during a galaxy's life; it also increases due to star formation and mergers, but by a smaller percentage, as confirmed both by observational evidence, showing that up to $z \sim 1$ the mass function evolves only mildly (see Pozzetti et al. 2007, and references therein), and by numerical simulations, (De Lucia et al. 2006). As a consequence, the selection of mass-limited samples eases the task of tracing the same population of galaxies in the different redshift bins explored. In the subsequent Sections, we will investigate the impact of the use of mass-selected samples on our analysis.

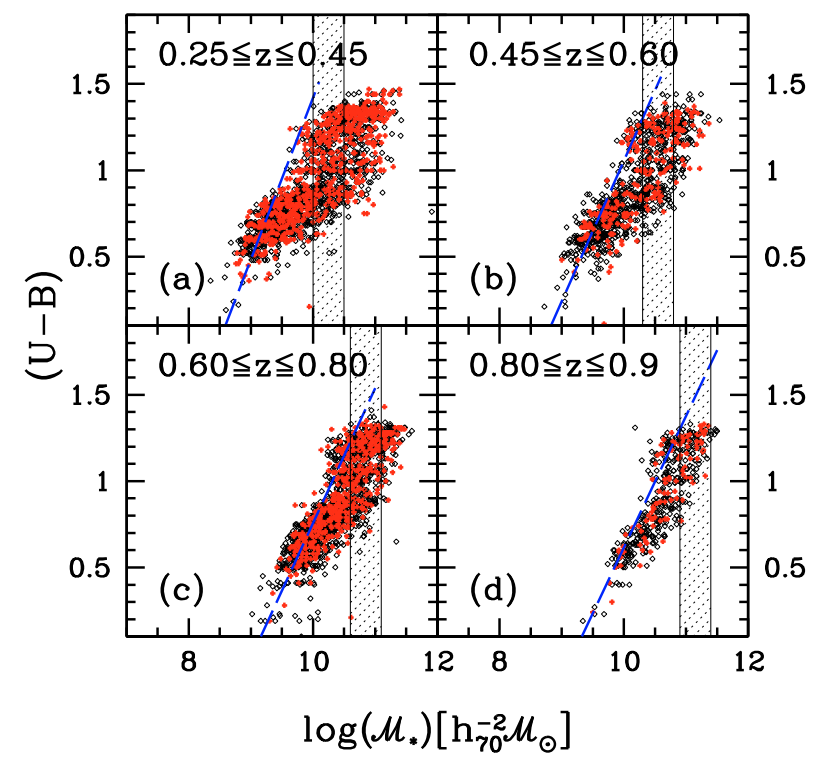

Fig. 10. Rest-frame $(U-B)$ colors plotted versus galaxy stellar mass in solar mass units for the samples defined in Table 1. On top of each panel, we write the redshift bins considered. For each panel, only galaxies contained in the corresponding volume-limited sample have been plotted (sample I to sample IV). The points in red show galaxies located in groups, while those in black correspond to the total population of galaxies. The blue dashed line in each panel indicates the colordependent $85 \%$ completeness mass-limit (see text for more details on how it is computed). The shaded area indicates the mass bins that we used in our analysis, and its lower boundary in mass equals the limiting mass above which all galaxies, irrespective of their color, are observed in our flux-limited survey.

\section{Defining stellar-mass, volume-limited samples}

To construct volume-limited, stellar-mass selected samples, we followed a simple approach. For each of the four redshift bins adopted in the previous analysis, we estimated the limiting mass at which even the oldest/reddest galaxies (i.e., those with the maximum possible stellar mass-to-light ratio) would be observable given the magnitude limit of our survey.

To estimate this limiting mass, we first proceeded by calculating the limiting stellar mass of each galaxy, i.e., the stellar mass it would have, at its spectroscopic redshift, if its apparent magnitude were equal to the limiting magnitude of our survey $\left(I_{A B}=22.5\right)$. We then used these estimated limiting masses to define, in bins of $(U-B)$ rest-frame colors for each redshift bin, the mass $\mathcal{M}_{\text {cut-off }}$ below which $85 \%$ of galaxies of that color lie. The value of $\mathcal{M}_{\text {cut-off }}$ for the reddest galaxies in each redshift bin is the one that we use as limiting mass.

Figure 10 shows the distribution of $(U-B)$ colors versus stellar masses for each of the volume-limited samples defined in Table 1. The points in red show galaxies located in groups, while those in black correspond to the total population of galaxies. The blue, dashed line in each panel indicates the fit to the values of the color-dependent $85 \%$ completeness mass-limit, estimated as described above. The lower boundary to the shaded rectangular area in each panel indicates the limiting mass above which all galaxies, even those redder in colors, are observed in our magnitude-limited survey. Figure 10 confirms that adopting mass volume-limited samples rejects a large number of lowermass, bright, blue galaxies, which were included in $B$-band luminosity, volume-limited samples. 
Table 3. Summary of the four mass volume-limited data samples; Mass is in units of $\log \left(\mathcal{M}_{*} /\left(\mathcal{M}_{\odot} \times h_{70}^{-2}\right)\right)$.

\begin{tabular}{ccccc}
\hline \hline & Sample M-I & Sample M-II & Sample M-III & Sample M-IV \\
stellar mass range & Mass $\geq 10.0$ & Mass $\geq 10.3$ & Mass $\geq 10.6$ & Mass $\geq 10.9$ \\
$z$ range & $0.1 \leq z \leq 0.45$ & $0.1 \leq z \leq 0.6$ & $0.1 \leq z \leq 0.8$ & $0.1 \leq z \leq 0.9$ \\
\hline All galaxies & 883 & 914 & 1033 & 491 \\
Isolated galaxies & 119 & 141 & 131 & 55 \\
Group galaxies I & 386 & 355 & 330 & 137 \\
Group galaxies II & 155 & 165 & 230 & 137 \\
\hline
\end{tabular}

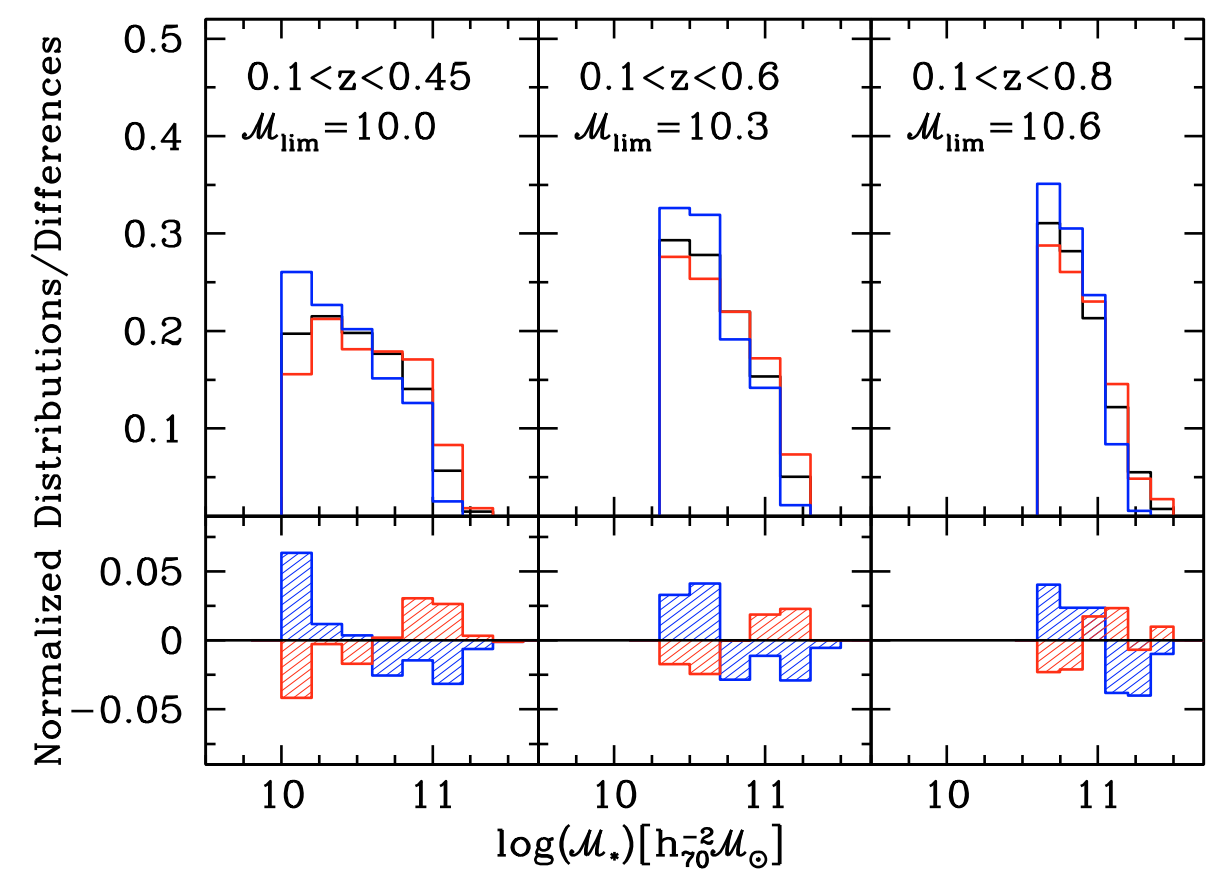

Fig. 11. Top panels: normalized histograms of galaxy mass for different galaxy populations. Isolated galaxies are in blue, group galaxies are in red and black galaxies are the full sample. Labels on top indicate the redshift bin considered and the cut-off mass adopted. Bottom panels: histogram of normalized differences with respect to the full galaxy sample of the group population (shaded in red) and the isolated galaxy population (shaded in blue). With respect to the whole galaxy population there is a visible and statistically significant excess of low/high mass galaxies in the isolated/group galaxy sample.
For each of the mass volume-limited samples Table 3 summarizes its lower mass limit and the number of galaxies contained in both the full galaxy sample and isolated/group galaxy samples.

Comparing the numbers in Table 3 with those in Table 1, it is clear that the samples that become more depleted moving from a luminosity to a mass selection are those of isolated galaxies. On average, these samples experience a decrease in number by a factor of $\sim 3$. The group samples, instead, are at most just halved. The blue low-mass galaxies that are excluded when moving from luminosity to mass limited samples are a larger fractions of galaxies residing in low-density environments. It is natural to conclude that at least part of the strong trends of $F_{\text {blue }}$ as a function of environment observed in our volume-limited samples are driven by the large population of lower mass, bright blue galaxies for which we miss the redder, equally low mass, counterparts (see De Propris et al. 2003, for a similar suggestion). In other words, the trends that we witness in Fig. 7 are at least partly due to the bias in $B$-band magnitudes volume-limited samples against red, low-mass galaxies, which are too faint to be included by the adopted luminosity cut-off.

It remains to be seen whether these trends are still observed when adopting mass volume-limited samples for the analysis, or whether mass is all that is needed for predicting galaxy colors, irrespective of environment, a possibility still compatible with our results until now. Such a possibility is the one expected in simple, pure nature, galaxy formation models, where the characteristics of a galaxy (e.g., colors, spin) are primarily determined by the mass of the dark matter halo in which it resides, which is in turn closely related to the galaxy stellar mass on one side, and to the density field on a $\sim 1 \mathrm{Mpc}$ scale on the other side (see, e.g., Cooray \& Sheth 2002). In this framework, the color segregation just mirrors the change in the distribution of galaxy stellar masses as a function of environment.

\subsection{Mass segregation in groups up to $z \sim 1$}

The variation in the galaxy stellar mass function between different environments has been observed in the local Universe (see, e.g., Baldry et al. 2006), and, at higher redshifts, in DEEP2 data (Bundy et al. 2006), in VVDS data (Scodeggio et al. 2009), in COSMOS data (Scoville et al. 2007), and in zCOSMOS 10K-sample data (Bolzonella et al. 2009). In this section, we check whether mass segregation is detectable using our group, field, and isolated galaxy samples.

Figure 11 shows in its top panels the normalized histograms of the mass distribution for the first three mass volume-limited samples of Table 3, plotted in red, blue and black for the group, isolated, and all galaxy samples, respectively. The bottom panels show, shaded in red, the difference between the group and all-galaxy normalized histograms, and shaded in blue the difference between the isolated and all-galaxy normalized histograms. There is a visible excess of both low-mass galaxies in the isolated galaxy sample and high-mass galaxies in the group galaxy sample, and the significance of this trend, estimated using a K-S test, is always at least $\sim 2.3 \sigma$ or more for all mass/redshift ranges considered. For sample M-IV of Table 3, there is no significant difference between the mass distributions of isolated and 
A. Iovino et al.: The zCOSMOS redshift survey: how group environment alters global downsizing trends

Table 4. Summary of the four mass volume-limited data samples; Mass is in units of $\log \left(\mathcal{M}_{*} /\left(\mathcal{M}_{\odot} \times h_{70}^{-2}\right)\right)$.

\begin{tabular}{ccccc}
\hline \hline & Sample MM-I & Sample MM-II & Sample MM-III & Sample MM-IV \\
stellar mass range & $10.0 \leq$ Mass $\leq 10.5$ & $10.3 \leq$ Mass $\leq 10.8$ & $10.6 \leq$ Mass $\leq 11.1$ & $10.9 \leq$ Mass $\leq 11.4$ \\
$z$ range & $0.1 \leq z \leq 0.45$ & $0.1 \leq z \leq 0.6$ & $0.1 \leq z \leq 0.8$ & $0.1 \leq z \leq 0.9$ \\
\hline All galaxies & 437 & 617 & 885 & 477 \\
Isolated galaxies & 64 & 101 & 117 & 45 \\
Group galaxies I & 174 & 221 & 330 & 132 \\
Group galaxies II & 56 & 95 & 187 & 132 \\
\hline
\end{tabular}

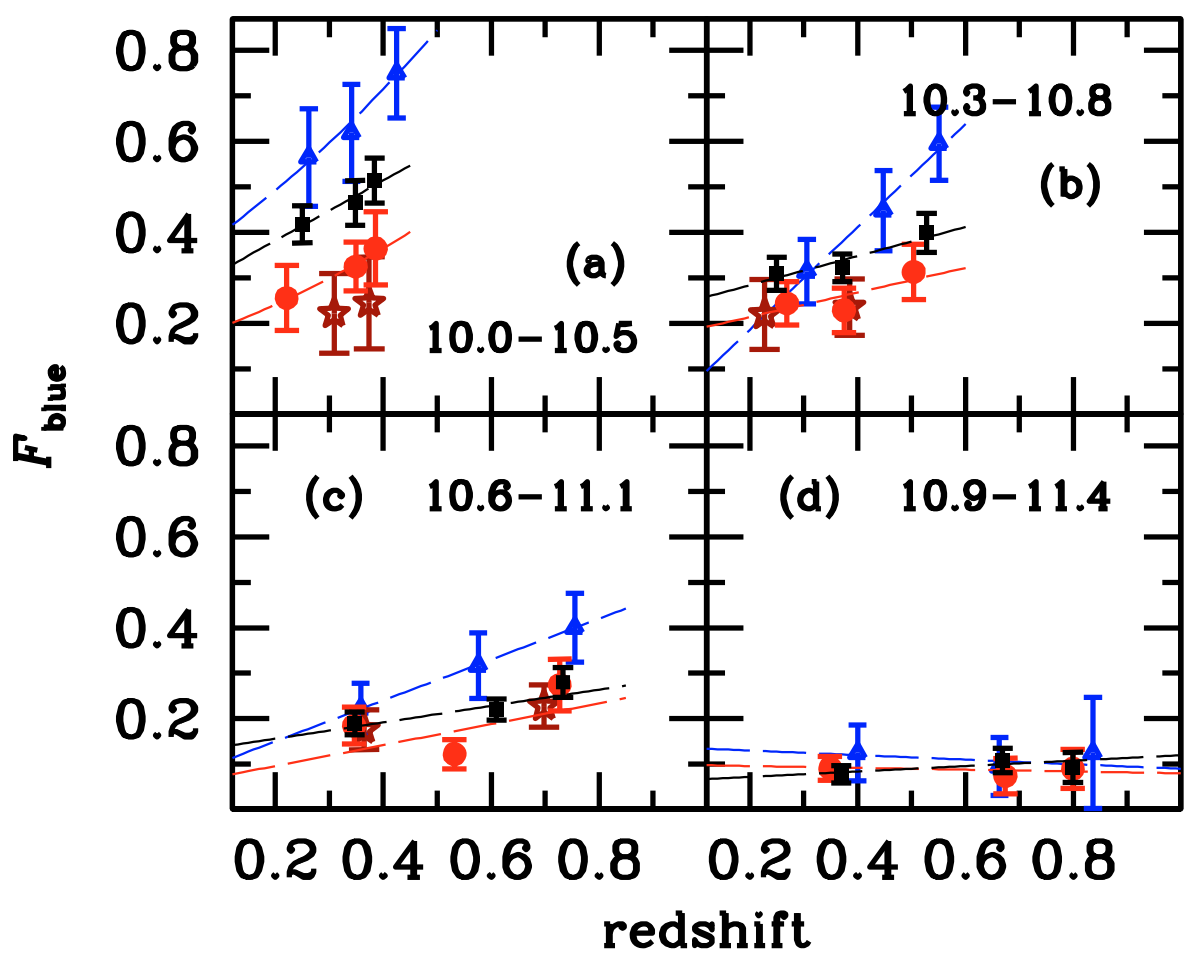

Fig. 12. The four panels show $F_{\text {blue }}$ as a function of redshift for each of the different mass limited samples defined in Table 3, as indicated by the labels. Red circles refer to group galaxies, blue triangles to isolated galaxies, and black squares to the total galaxy population. Brown stars are those corresponding, for each of the mass limited sample considered, to the population of galaxies in groups with at least two members in sample IV. While for the lowest mass bin explored there is still a significant residual difference in color as a function of environment, such difference progressively disappears moving to higher masses. group galaxies, a result possibly caused by both the lower number statistic and the narrower mass range considered. We therefore have not plotted the corresponding histograms.

Given these differences in the mass distribution in different environments, we need to define mass bins that are narrow enough for mass segregation to become negligible before we can disentangle the mass/environment influence in determining galaxy colors. Only in this way shall we be able to check if environment has truly some influence on galaxy colors other than being the by-product of mass segregation. This is the approach we adopt in the following section, using as mass bins those indicated by the gray shaded areas in Fig. 10. A K-S test applied to the mass distribution within these bins confirms that there is no residual significant difference in mass among galaxies located in different environments.

\subsection{Blue fraction as a function of galaxy mass and environment up to $z \sim 1$}

We explore how $F_{\text {blue }}$ changes as a function of environment in bins of mass volume-limited samples. For our analysis, we use the logarithmic mass bins shown by the shaded rectangles in Fig. 10, whose number of galaxies are listed in Table 4. Because of the large reduction in our sample when adopting mass volume-limited samples, we used bins partially overlapping in mass, a choice dictated by the desire to have a sufficient number of galaxies in each bin such that our findings could be deemed statistically significant. As a consequence, the results shown for the various mass bins are not completely independent. Needless to say, the completion of zCOSMOS bright will enable a much more detailed analysis.

Figure 12 shows the fraction of blue galaxies as a function of redshift in the four samples and in the three galaxy samples listed in Table 4. In each of the four panels, the red circles show $F_{\text {blue }}$ for group galaxies, while the black squares and the blue triangles show the same quantity for field and isolated galaxies, respectively. The labels in each panel indicate the mass range under inspection.

The first information that this plot conveys is that color segregation is still present in the lowest mass bin explored, the one shown in panel (a), while in the intermediate-mass bins, i.e., in panels (b) and (c), is barely detectable and only at the highest limits of the redshift ranges explored. For the highest mass bin, in panel (d), there is no hint of a color segregation up to $z \sim 1$ and no evolution with redshift is detectable.

We parametrized the evolution in $F_{\text {blue }}$ with a fit of the form $F_{\text {blue }} \propto(1+z)^{\beta}$, and the results are indicated by the dashed lines in Fig. 12. When dealing with samples defined by bins in mass, however, at odds with what we discussed in Sect. 8.2, the dashed lines obtained from the fit to the data points seem, if anything, to indicate that the relative differences of $F_{\text {blue }}$ between group, field, and isolated galaxy samples progressively disappear moving from high to low redshift. 
Table 5. Summary of fits results for mass bins of Table 4 Mass is in units of $\log \left(\mathcal{M}_{*} /\left(\mathcal{M}_{\odot} \times h_{70}^{-2}\right)\right)$.

\begin{tabular}{ccccccccc}
\hline \hline & \multicolumn{3}{c}{ Sample MM-I } & \multicolumn{2}{c}{ Sample MM-II } & \multicolumn{2}{c}{ Sample MM-III } & \multicolumn{2}{c}{ Sample MM-IV } \\
stellar mass range & \multicolumn{2}{c}{$10.0 \leq$ Mass $\leq 10.5$} & \multicolumn{2}{c}{$10.3 \leq$ Mass $\leq 10.8$} & \multicolumn{2}{c}{$10.6 \leq$ Mass $\leq 11.1$} & \multicolumn{2}{c}{$10.9 \leq$ Mass $\leq 11.4$} \\
$z$ range & \multicolumn{2}{c}{$0.1 \leq z \leq 0.45$} & \multicolumn{2}{c}{$0.1 \leq z \leq 0.6$} & \multicolumn{2}{c}{$0.1 \leq z \leq 0.8$} & $0.1 \leq z \leq 1.0$ \\
\hline & $F_{\text {blue }}(z=0)$ & $\beta$ & $F_{\text {blue }}(z=0)$ & $\beta$ & $F_{\text {blue }}(z=0)$ & $\beta$ & $F_{\text {blue }}(z=0)$ & $\beta$ \\
All galaxies & $0.27 \pm 0.10$ & $1.9 \pm 1.2$ & $0.22 \pm 0.06$ & $1.3 \pm 0.8$ & $0.12 \pm 0.04$ & $1.4 \pm 0.7$ & $0.06 \pm 0.04$ & $1.0 \pm 1.2$ \\
Isolated galaxies & $0.32 \pm 0.18$ & $2.4 \pm 1.7$ & $0.11 \pm 0.06$ & $3.7 \pm 1.4$ & $0.11 \pm 0.06$ & $2.3 \pm 1.1$ & $0.14 \pm 0.20$ & $-0.5 \pm 2.6$ \\
Group galaxies I & $0.15 \pm 0.11$ & $2.7 \pm 2.4$ & $0.16 \pm 0.08$ & $1.5 \pm 1.5$ & $0.10 \pm 0.05$ & $1.6 \pm 1.1$ & $0.10 \pm 0.07$ & $-0.3 \pm 1.5$ \\
Group galaxies II & $0.13 \pm 1.18$ & $2.0 \pm 9.9$ & $0.20 \pm 0.19$ & $0.6 \pm 3.1$ & $0.12 \pm 0.08$ & $1.2 \pm 1.3$ & $0.10 \pm 0.06$ & $-0.3 \pm 1.5$ \\
\hline
\end{tabular}

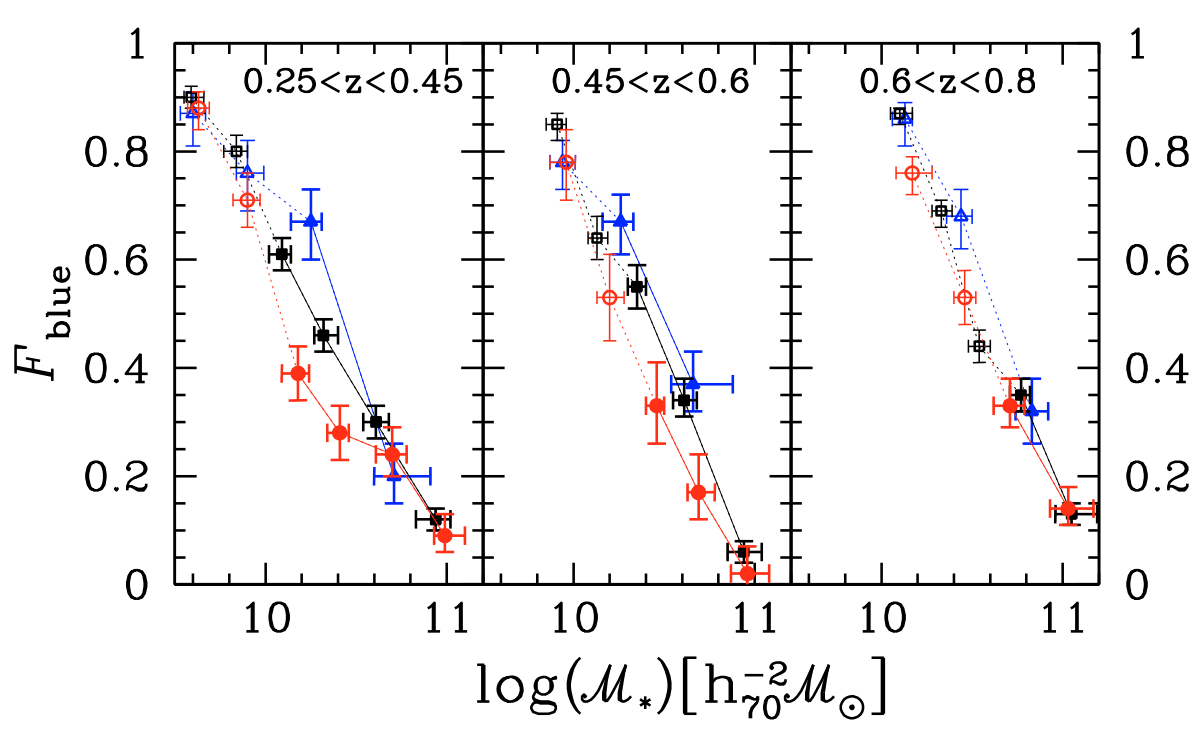

Fig. 13. The three panels show, in the redshift bin indicated by the top labels, $F_{\text {blue }}$ as a function of galaxy stellar mass. Red circles refer to group galaxies, blue triangles to isolated galaxies, while black squares to the total galaxy population. Error bars along the $y$-axis are those obtained by bootstrap, and those along the $x$-axis indicates the inter-quartiles ranges of the mass distribution in the bin under scrutiny.
One can imagine a time when, irrespective of the environment considered, most galaxies in each of the mass bin ranges explored, reside on the red sequence, having exhausted their fuel for star formation, while the blue cloud becomes more or less empty. This seems to be already the case for the highest mass bin in our plot. Panel (d) indicates that the majority of red-sequence galaxies in the mass range $10.9 \leq \log \left(\mathcal{M}_{*} / \mathcal{M}_{\odot}\right) \leq 11.4$, were already in place, irrespective of the environment, at the highest redshift bin we can explore $(z \sim 0.9)$.

In contrast, for the lower mass bins explored, our data display a significant decrease in $F_{\text {blue }}$ between high and low redshifts. Extrapolating the observed trends further back in time up to $z \sim 1$, one can speculate that there must have been a time when most galaxies resided in the blue cloud, irrespective of their environment. Panel (a) of Fig. 12 clearly suggests that the time when blue galaxies were in the majority has ended earlier for galaxies in groups than for those in field or isolated galaxies, and a similar trend is present, albeit at a far lower significance, also for galaxies in panels (b) and (c).

Unfortunately, as shown by Table 5 , the error bars in the slopes of the fit are quite large and it is difficult to draw definitive conclusions on the fractional rate of change in $F_{\text {blue }}$ for the different environments of each mass bin considered. In each redshift bin, all the values obtained for $\beta$ are compatible with each other for the three environments considered, given their large error bars.

In parallel with the analysis completed in Sect. 5 for the mass volume-limited samples we proceeded to plot in Fig. 12 results for galaxies residing in groups homogeneous in richness across the different panels. We again used the sample of groups with two or more members in the brighter absolute magnitude cutoff sample, and then repeated our measurements of $F_{\text {blue }}$ for the group members satisfying the mass-bins limits. The numbers of the galaxy group samples defined in this way are those indicated by the entry Group galaxies II in Table 4.

With the possible exception of panel (a), moving to richer groups does not seem to affect significantly the value of $F_{\text {blue }}$. This result is consistent with the previous one: only for galaxies of lower stellar masses do we still see color segregation as a function of environment and therefore only for these masses can we expect to see a significant dependence of $F_{\text {blue }}$ on group richness.

An interesting trend suggested by Fig. 12 is that for more massive galaxies the predominance of the redder-color galaxy population started earlier in cosmic time than for lower mass galaxies. We decided to investigate this trend directly by plotting at fixed redshift $F_{\text {blue }}$ as a function of mass.

The results are shown in Fig. 13. The label on each of the three panels shows the redshift ranges adopted, while the color code is, as usual, blue, red, and black for isolated, group and field galaxies, respectively.

We adopted the following three redshift bins where to perform this analysis [0.25:0.45], [0.45:0.60] and [0.60:0.80]. For these bins, we had already defined complete mass limited samples as listed in Table 3. However, to increase the range of masses probed in each redshift bin we decided to extend the analysis down to masses where, according to the procedure described in Sect. 8, we had in the redshift bin considered a completeness lower than $85 \%$ for the reddest galaxies of our sample. Obviously such a strategy can be adopted only if one is sure that a representative (in color) sample of the lower mass galaxies under scrutiny is observed in a large fraction of the volume under consideration, so that it can be statistically reconstructed, e.g., applying a correction using the $V / V_{\max }$ technique. We 
therefore lowered our mass limit to masses such that the completeness even for the reddest galaxies was always around $100 \%$ at the lowest limit of the redshift bin considered. We then weighted each observed galaxy with its corresponding volume correction, estimated as the ratio of the volume contained within the $\left[z_{\min }: z_{\max }\right]$ bin and the actual volume up to which the galaxy can be observed within the survey $I_{A B} \leq 22.5$ selection.

Filled points in Fig. 13 are those referring to bins in masses where we are complete, while empty points refer to the lower masses bins where the $V / V_{\max }$ corrections discussed above have been applied.

In the $x$-axis we plot the median mass value for each mass bin, while in the $y$-axis is the value of $F_{\text {blue }}$ for the same bin. The error-bars along the $y$-axis are those obtained with bootstrap analysis, and the ones along the $x$-axis indicate the inter-quartiles ranges of the mass distribution within the mass bin considered. The choice of the mass bins is somewhat arbitrary, as for the isolated galaxies sample we were forced to split the sample in fewer bins for statistical reasons.

The trends displayed in the lowest redshift bin well agree qualitatively with similar trends detected at $z \sim 0$ (e.g., Kauffmann et al. 2004; Baldry et al. 2006), showing a clear dependence of $F_{\text {blue }}$ both on mass and on environment. However, there are some new interesting points, obserbed thanks to the unprecedented wide redshift/mass ranges covered by our dataset.

On one hand, at all redshift bins more massive galaxies always display a lower $F_{\text {blue }}$, near to zero values, irrespective of the environment they live in, while for lower mass galaxies the value of $F_{\text {blue }}$ raises towards unity, again irrespective of the environment they live in. Therefore, in each redshift range, and more clearly in the first two ones plotted, where the mass coverage is wider, we witness the presence of a progressive saturation of $F_{\text {blue }}$ towards high/low values at the extremes of the mass ranges studied. However, there is a restricted range of masses for which the color of galaxies show a visible dependence on environment. This mass range is the one where both sides of the bimodal distribution of galaxy colors are well populated and we can detect a considerable environment dependent variation of $F_{\text {blue }}$. This result echoes a similar one obtained by Kauffmann et al. (2004) in the local Universe.

On the other hand, moving from lower to higher redshifts we witness a progressive increase of $F_{\text {blue }}$ for each mass bin, with the possible exception of the highest masses, as already observed in the previous section. Such decrease of $F_{\text {blue }}$ as cosmic time goes by seem to be accompanied by a progressive opening along the $x$-axis of the difference between the different environments, most prominent in the mass ranges for which $F_{\text {blue }} \sim 0.5$.

\subsection{Detection of the possible signature of environmental effects}

It is interesting to quantify the trend discussed at the end of the previous section using a simple parameter: the value, for each mass bin and environment, of the redshift when $F_{\text {blue }}=0.5$. We can call this quantity $t_{50-50}$ to indicate that it corresponds to the time when the galaxies in the environment and mass bin considered were equally partitioned between blue and red colors. Although obtained through a slightly different type of analysis, this quantity is equivalent to the transitional mass $m_{\text {tr }}$ identified by various authors, both in the low redshift regime by Baldry et al. (2004) and Kauffmann et al. (2004) and at higher redshifts by Bundy et al. (2006) and for the 10K-sample by Bolzonella et al. (2009).

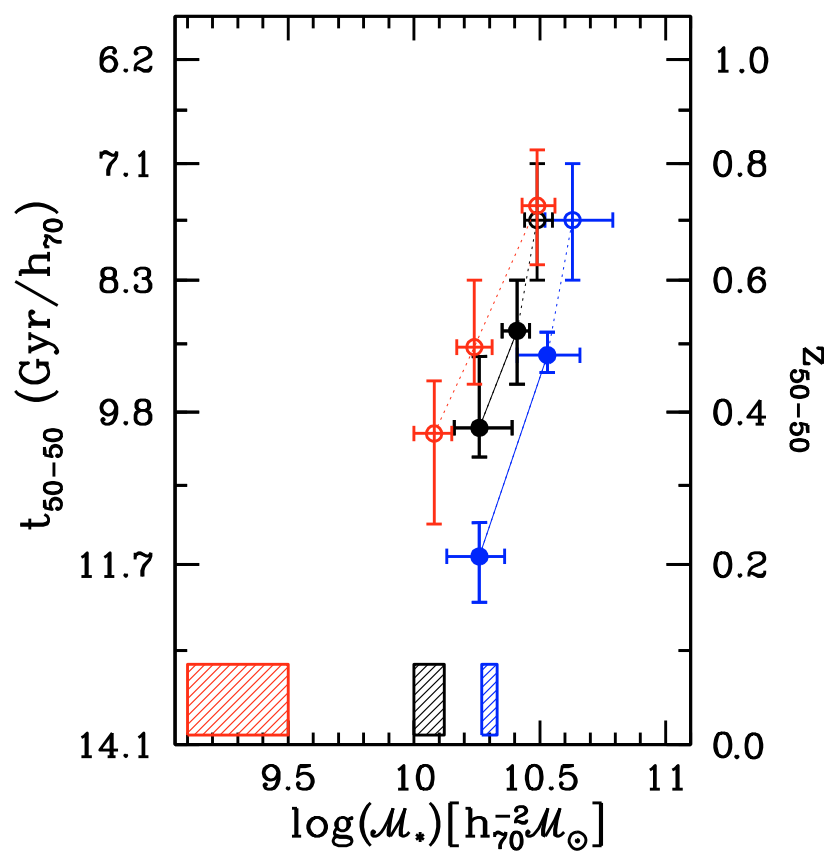

Fig. 14. The time $t_{50-50}$, at which $F_{\text {blue }} \sim 0.5$, is plotted as a function of galaxy stellar mass. The left scale is in units of cosmic time, in Gyr, while the right scale is in redshift. The triangles, circles, and squares refer to the sample of isolated, group and field galaxies, respectively. Filled points are those corresponding to bins in masses where we are complete, while empty points refer to the lower mass bins, where the $V / V_{\max }$ corrections are needed. The shaded boxes have been obtained from Baldry et al. (2006). See text for more details.

Figure 14 shows the value of $t_{50-50}$, expressed in units of time on the left-hand scale and redshift on the right-hand scale, for different galaxy stellar masses. The triangles, circles, and squares refer to the sample of isolated, group and field galaxies, respectively. Filled points are estimated from Fig. 12 using the fits to the points plotted in each mass bin as shown in Table 5. The values obtained in this way for $t_{50-50}$ do not need any incompleteness correction, since they are observed directly in our $10 \mathrm{~K}$-sample in a mass range where we are complete, but they cover only a limited range of mass and environments. Empty points are obtained using a $V / V_{\max }$ correction and Fig. 13. These values for $t_{50-50}$ are therefore more uncertain, since they are based on incompleteness corrections.

For filled points, the error bars along the $x$-axis link the upper and lower quartiles of the mass distribution in the mass bin considered, while the error bars along the $y$-axis are obtained by the rms of the value of $t_{50-50}$ as obtained by bootstrapping the sample of galaxies that enters in the corresponding panels of Fig. 12. For empty points, the error bars along the $y$-axis indicate the redshift bin where the value of $t_{50-50}$ was estimated, while the error bars along the $x$-axis show the upper and lower interval for the masses as obtained from the error bars in Fig. 13.

For the sake of comparison, we have added boxes indicating masses for which $t_{50-50} \sim 0.05$, as obtained from the relationship between mass, galaxy colors, and environment in the local Universe determined in Baldry et al. (2006). These values are only indicative and extracted from their Fig. 11, panel b, where curves of the fraction of red galaxies versus stellar mass are shown in 12 different bins of galaxy density. We choose to use as representative of the global population the two central curves of their plot, corresponding to $-0.2 \leq \log (\Sigma) \leq 0.2$, while for isolated/group galaxies in the local Universe we used the curves 
covering densities $-0.8 \leq \log (\Sigma) \leq-0.4$ and $+0.4 \leq \log (\Sigma) \leq$ +0.8 . This last choice was made considering that our total span in densities is not as wide as theirs: the bulk of the galaxy population in our groups/isolated galaxies is located in regions that are roughly a factor of 3 above/below median densities (see Fig. 15, panel a in Kovac et al. 2009b, and a similar plot for our isolated galaxy population).

The vertical size of the boxes plotted in Fig. 14 corresponds to the redshift range of the sample used in Baldry et al. (2006), while the mass range corresponding to the horizontal box size corresponds to the range of galaxy stellar mass values where the fraction of galaxies in the red sequence equals 0.5 for the three different environments defined by the curves mentioned above. It should be noticed that the masses in Baldry et al. (2006) were estimated using a Kroupa (2001) initial mass function and recurrent bursts of star formation superimposed to continuous models of star formation. As a consequence the Baldry et al. (2006) masses have a systematically offset towards higher values with respect to our masses by a factor that can be as high as 0.15 dex, possibly explaining the slight offset of local points with respect to the trends displayed by our high- $z$ points.

Figure 14 highlights the main result of our paper. The first visible trend is that as cosmic time goes by the typical mass at which the galaxy population is equally partitioned between red and blue galaxies moves progressively to lower values. This is another way of expressing the well-known downsizing pattern observed in galaxy population evolution. In our plot the decrease in galaxy $K$-band luminosity with decreasing redshift of galaxies dominated by star formation, as originally reported by Cowie et al. (1996), translates into a progressive increase of $t_{50-50}$ when considering galaxies of lower masses.

But this global behavior displays differences depending on the subset of the galaxy population we are considering. A consistent trend emerges, despite the large error bars: for each mass considered $t_{50-50}$ is progressively delayed moving from groups to the field and to the isolated galaxy population. In other words the downsizing for the galaxy population is further modulated by the environment: galaxies located in more massive halos (groups) become red earlier in cosmic time, a trend that shows again a downsizing behavior on the larger scales now considered. The trends displayed by our data well match those observed in the local Universe by Baldry et al. (2006).

Last but not least, another interesting trend suggested by Fig. 14 is the convergence, visible at higher masses, of the value of $t_{50-50}$, irrespective of the environment considered. We are aware that this interpretation is plagued by uncertainties, as for these high masses the redshifts are correspondingly higher and those most affected by various incompleteness/contamination effects. Two possible biases can be at play at higher redshifts: the progressive degradation in the efficiency of the group/isolated galaxies algorithms and the progressive incompleteness towards the red galaxy population. Both biases act in the direction of reducing the differences between the color properties of the galactic populations we are studying. However we expect that these two biases are minimal, as discussed at length in the previous sections.

One should also consider that for galaxies residing in more extreme density regimes, as those represented by rich cluster cores and not observed in our sample, there could be a residual difference even at redshift $\sim 1$ from the general galaxy population (see, e.g., Tanaka et al. 2008). As already suggested in the introduction, however, the physical mechanisms responsible for these differences are presumably not the same as those at play in the group environment. Our results parallel those obtained, although by a different kind of analysis, by Bolzonella et al. (2009), and those shown for galaxies morphologies by Kovac et al. (2009b).

We note that the evidence we presented, i.e., the faster shutdown of star formation in group environment - as the color transition from blue to red galaxy can broadly be interpreted - cannot be interpreted utilizing only ab initio/internal mechanisms, and was obtained thanks to the unprecedented wide redshift, mass and environment ranges covered by our survey.

The different value of $t_{50-50}$ for galaxies of different stellar masses, irrespective of environment, can be explained by resorting to internally driven mechanisms shutting-down star formation. The presence of an active galactic nucleus (AGN) feedback and shock-heating physics can be enough to explain the anti-hierarchical nature of the relation between stellar mass and stellar age of galaxies, because these mechanisms can be more efficient in more massive galaxies (see Birnboim \& Dekel 2003; Bower et al. 2006; Bundy et al. 2006; Croton et al. 2006; Cattaneo et al. 2008).

In a similar way, the detection of an offset in the value of $t_{50-50}$ between samples of group/isolated galaxies at fixed stellar mass does not necessarily imply that nurture mechanisms are at work. It could be explained by a different time of assembly of galaxies in haloes of different masses, a nature mechanism that results in a more evolved galaxy population in groups and clusters, at fixed stellar mass, in a given redshift bin (see Gao et al. 2005; Balogh et al. 2007).

In contrast, the trend suggested by Fig. 14 indicates that the migration of galaxies from the blue cloud to the red sequence is a process more efficient/faster in groups than in isolated/field galaxies, and is therefore the signature of environmental processes at play in groups in shaping galaxy evolution.

Interestingly, such mechanisms seem to become progressively more relevant moving to lower galaxy stellar masses, while they seem to be irrelevant to galaxies of higher stellar masses, at least in the redshift range we explored (see also Bolzonella et al. 2009). We can therefore distinguish between two different channels for the production of red galaxies, corresponding respectively, to use a common nomenclature, to nature red galaxies and nurture red galaxies. Our results suggest that galaxies of masses $\approx 10.8$ solar in logarithmic scale are already in place by $z \sim 1$ and their origin could be due primarily to socalled nature/internal mechanisms, as no strong environmental dependency is visible up to $z \sim 1$. In contrast, for masses below this value and at redshifts lower than $z \sim 1$, we witness the emergence in groups of an additional contribution of red galaxies. This is what we can call nurture red galaxies: galaxies that deviate slightly from the trend of the downsizing scenario as displayed by the global galaxy population. This nurture population is the one responsible for the earlier value of $t_{50-50}$ in groups, and its importance grows as cosmic time goes by, causing the steady growth in the difference of $t_{50-50}$ moving to lower galaxy masses.

There are various mechanisms that occur in groups and that are more efficient for less massive galaxies, including the gradual cessation of star formation induced by gentle gas stripping and starvation by a diffuse intragroup medium, or by slow group-scale harassment (see, e.g., Larson et al. 1980; Moore et al. 1999; Gnedin 2003; Roediger \& Hensler 2005; Kawata \& Mulchaey 2008). These mechanisms could be natural candidates for explaining the trends we observe. Their increasing importance after $z \sim 1$ most probably mirrors the progressive emergence of structures, as predicted by hierarchical clustering growth scenario, where such mechanisms can effectively take place. 


\section{Summary and conclusions}

Taking advantage of the large coverage both of redshift and galaxy/group properties in the $10 \mathrm{~K}$ galaxy/groups catalogue, we revisited of the blueing of the galaxy population in groups toward higher redshift, originally observed by Butcher \& Oemler (1978), gaining some interesting new insights, that can be summarized as follows.

1. We have showed that using rest-frame $B$-band volumelimited samples, the group galaxy population becomes bluer as redshift increases, but maintains a systematic difference with respect to the global galaxy population, and an even larger difference with respect to the isolated galaxy population. Superimposed on this global effect, we detected additional trends as a function of both galaxy $B$-band rest-frame luminosity and group properties. More luminous galaxies exhibit stronger variations in $F_{\text {blue }}$ among group, field, and isolated environments and groups richer or with higher $\sigma$ show a lower $F_{\text {blue }}$.

2. The difference between the three different environments increases between high and low redshift. At the highest redshift bin explored $(z \sim 1)$, there is a small but still significant difference in $F_{\text {blue }}$ among group,field, and isolated samples. This gradual increase in the $F_{\text {blue }}$ difference with cosmic time is a clear signature of an environmental dependence, but not necessarily of the existence of environmental effects at work. It could be the result of an ab initio bias that favors later formation of lower-mass galaxies in lower density environments, causing the delayed and more efficient replenishing of the blue cloud in lower density environments.

3. Moving to mass-selected samples, a necessary step in clarifying the key mechanisms in determining the relationship observed using luminosity-selected samples, allows us to realize almost immediately that at least part of the strong trends observed when using rest-frame evolving $B$-band volumelimited samples are caused by the large population of lower mass, bright blue galaxies for which we miss the redder, equally low mass, counterparts. In other words, the biased view imposed by the $B$-band luminosity selection amplifies the findings obtained using B-band volume-limited samples.

4. Another effect has to be taken into consideration if one wants to disentangle the mass and environment influence on galaxy colors. The existence of different mass functions in different environments (see Bolzonella et al. 2009) forces us to work in mass bins narrow enough so that any color segregation cannot be attributed simply to the different mass distribution.

5. The first outcome of this careful analysis is that there is still a significant residual difference in color as a function of environment only for the lowest mass bin explored (Mass $\leq 10.6$, solar masses in logarithmic scale), while this difference progressively disappears moving to higher masses.

6. By using a $V / V_{\max }$ correction, we can extend our analysis to lower masses, witnessing, in all the redshift range we explore, the presence of progressive saturation of $F_{\text {blue }}$ towards high/low values at the extremes of the mass ranges studied. At each redshift, there is a restricted range of masses for which the color of galaxies show a visible dependence on environment, and as cosmic time increases the typical mass at which the galaxy population is equally partitioned between red and blue galaxies moves progressively to lower values. This pattern, consistent with the well known downsizing pattern observed in galaxy population evolution, is further modulated by environment: galaxies located in more massive halos (groups) become red earlier in cosmic time.
7. Finally our most interesting finding is that there is evidence that the color transition from blue to red galaxies seems to be faster in groups as cosmic time increases. In other words, we seem to witness the slow emergence of an environmen$\mathrm{tal} /$ nurture effect on galaxy evolution, which causes the faster migration of galaxies from the blue cloud to the red sequence in groups (with respect to isolated/field galaxies) and effect that becomes more relevant moving from higher to lower galaxy stellar masses (see also Kovac et al. 2009b, for a parallel analysis using galaxy morphologies).

8. Our results suggest that galaxies of Mass $\approx 10.8$ solar masses in logarithmic scale are already in place by $z \sim 1$ and their origin could be due primarily to so-called nature/internal mechanisms, since no strong environmental dependency is detectable up to $z \sim 1$.

9. In contrast, for masses below this value and at redshifts lower than $z \sim 1$ we witness the emergence of what we call nurture red galaxies: galaxies that slightly deviate from the trend of the downsizing scenario displayed by the global galaxy population and do more so as cosmic time progresses.

There are various mechanisms that occur in groups and are more efficient for less massive galaxies (gradual cessation of star formation induced by gentle gas stripping and starvation by a diffuse intragroup medium, or by slow group-scale harassment). These mechanisms could be the natural candidates to explain the trends observed after $z \sim 1$, a timing that could simply mirror that of the progressive emergence of structures where these mechanisms can effectively take place.

The completion of zCOSMOS bright, and subsequent availability of 20K-sample, will enable us to place on a more robust basis this result, which indicates that environment starts playing an active role in shaping galaxy evolution after $z \sim 1$.

Acknowledgements. We acknowledge support from an INAF contract PRIN2007/1.06.10.08 and an ASI grant ASI/COFIS/WP3110 I/026/07/0.

\section{References}

Abell, G. O. 1958, ApJS, 3, 211

Allington-Smith, J. R., Ellis, R., Zirbel, E. L., \& Oemler, A. J. 1993, ApJ, 404, 521

Andreon, S., \& Ettori, S. 1999, ApJ, 516, 647

Andreon, S., Lobo, C., \& Iovino, A. 2004, MNRAS, 349, 889

Andreon, S., Quintana, H., Tajer, M., Galaz, G., \& Surdej, J. 2006, MNRAS, 365,915

Baldry, I. K., Glazebrook, K., Brinkmann, J., et al. 2004, ApJ, 600, 681

Baldry, I. K., Balogh, M. L., Bower, R. G., et al. 2006, MNRAS, 373, 469

Balogh, M., Eke, V., Miller, C., et al. 2004a, MNRAS, 348, 1355

Balogh, M. L., Baldry, I. K., Nichol, R., et al. 2004b, ApJ, 615, L101

Balogh, M. L., Wilman, D., Henderson, R. D. E., et al. 2007, MNRAS, 374, 1169

Beers, T., Flynn, K., \& Gebhardt, K. 1990, ApJ, 100, 32

Berlind, A. A., Frieman, J., Weinberg, D. H., et al. 2006, ApJS, 167, 1

Birnboim, Y., \& Dekel, A. 2003, MNRAS, 345, 349

Biviano, A., Katgert, P., Mazure, A., et al. 1997, A\&A, 321, 84

Blanton, M. R., \& Berlind, A. A. 2007, ApJ, 664, 791

Blanton, M. R., Eisenstein, D., Hogg, D. W., \& Zehavi, I. 2006, ApJ, 645, 977

Bolzonella, M., Kovač, K., Pozzetti, L., et al. 2009, A\&A, submitted [arXiv: 0907.0013]

Bottini, D., Garilli, B., Maccagni, D., et al. 2005, PASP, 117, 996

Bower, R. G., Benson, A. J., Malbon, R., et al. 2006, MNRAS, 370, 645

Brüggen, M., \& De Lucia, G. 2008, MNRAS, 383, 1336

Bruzual, G., \& Charlot, S. 2003, MNRAS, 344, 1000

Bundy, K., Ellis, R. S., Conselice, C. J., et al. 2006, ApJ, 651, 120

Butcher, H., \& Oemler, Jr., A. 1978, ApJ, 219, 18

Butcher, H., \& Oemler, Jr., A. 1984, ApJ, 285, 426

Cattaneo, A., Dekel, A., Faber, S. M., \& Guiderdoni, B. 2008, MNRAS, 389, 567

Chabrier, G. 2003, ApJ, 586, L133

Coleman, G. D., Wu, C.-C., \& Weedman, D. W. 1980, ApJS, 43, 393

Cooper, M. C., Newman, J. A., Croton, D. J., et al. 2006, MNRAS, 370, 198 
Cooper, M. C., Newman, J. A., Coil, A. L., et al. 2007, MNRAS, 376, 1445 Cooray, A., \& Sheth, R. 2002, Phys. Rep., 372, 1

Cowie, L. L., Songaila, A., Hu, E. M. \& Cohen, J. G. 1996, AJ, 112, 839

Croton, D. J., Springel, V., White, S. D. M., et al. 2006, MNRAS, 365, 11

Cucciati, O., Iovino, A., Marinoni, C., et al. 2006, A\&A, 458, 39

Cucciati, O., Iovino, A., Kovač, K., et al. 2009a, A\&A, submitted

Cucciati, O., Marinoni, M., Iovino, A., et al. 2009b, A\&A, submitted

De Lucia, G., Springel, V., White, S. D. M., Croton, D., \& Kauffmann, G. 2006, MNRAS, 366, 499

De Lucia, G., Poggianti, B. M., Aragón-Salamanca, A., et al. 2007, MNRAS, 374, 809

De Propris, R., Stanford, S. A., Eisenhardt, P. R., \& Dickinson, M. 2003, ApJ, 598,20

De Propris, R., Colless, M., Peacock, J. A., et al. 2004, MNRAS, 351, 125

Dressler, A., \& Gunn, J. E. 1982, ApJ, 263, 533

Dressler, A., \& Gunn, J. E. 1992, ApJS, 78, 1

Eke, V. R., Baugh, C. M., Cole, S., et al. 2004, MNRAS, 348, 866

Ellingson, E., Lin, H., Yee, H. K. C., \& Carlberg, R. G. 2001, ApJ, 547, 609

Fabricant, D. G., McClintock, J. E., \& Bautz, M. W. 1991, ApJ, 381, 33

Fairley, B. W., Jones, L. R., Wake, D. A., et al. 2002, MNRAS, 330, 755

Feldmann, R., Carollo, C. M., Porciani, C., et al. 2006, MNRAS, 372, 565

Finoguenov, A., Guzzo, L., Hasinger, G., et al. 2007, ApJS, 172, 182

Gao, L., Springel, V., \& White, S. D. M. 2005, MNRAS, 363, L66

Gavazzi, G., Pierini, D., \& Boselli, A. 1996, A\&A, 312, 397

Gehrels, N. 1986, ApJ, 303, 336

Gerke, B. F., Newman, J. A., Davis, M., et al. 2005, ApJ, 625, 6

Gerke, B. F., Newman, J. A., Faber, S. M., et al. 2007, MNRAS, 376, 1425

Gnedin, O. Y. 2003, ApJ, 589, 752

Goto, T. 2005, MNRAS, 356, L6

Goto, T., Okamura, S., Yagi, M., et al. 2003, PASJ, 55, 739

Hopkins, A. M. 2004, ApJ, 615, 209

Huchra, J. P., \& Geller, M. J. 1982, ApJ, 257, 423

Ilbert, O., Capak, P., Salvato, M., et al. 2009, ApJ, 690, 1236

Kauffmann, G., White, S. D. M., Heckman, T. M., et al. 2004, MNRAS, 353, 713

Kawata, D., \& Mulchaey, J. S. 2008, ApJ, 672, L103

Kenney, J. D. P., van Gorkom, J. H., \& Vollmer, B. 2004, AJ, 127, 3361

Kinney, A. L., Calzetti, D., Bohlin, R. C., et al. 1996, ApJ, 467, 38

Kitzbichler, M. G., \& White, S. D. M. 2007, MNRAS, 376, 2

Knobel, C., Lilly, S. J., Iovino, A., et al. 2009, ApJ, 697, 1842

Kodama, T., \& Bower, R. G. 2001, MNRAS, 321, 18

Koekemoer, A. M., Aussel, H., Calzetti, D., et al. 2007, ApJS, 172, 196

Kovač, K., Lilly, S. J., Cucciati, O., et al. 2009a, ApJ, submitted [arXiv:0903.3409]

Kovac, K., Lilly, S. J., Knobel, C., et al. 2009b, ApJ, submitted [arXiv: 0909.2032]

Koyama, Y., Kodama, T., Tanaka, M., Shimasaku, K., \& Okamura, S. 2007, MNRAS, 382, 1719

Kroupa, P. 2001, MNRAS, 322, 231

Larson, R. B., Tinsley, B. M., \& Caldwell, C. N. 1980, ApJ, 237, 692

Lavery, R. J., \& Henry, J. P. 1986, ApJ, 304, L5

Lavery, R. J., \& Henry, J. P. 1988, ApJ, 330, 596

Le Fevre, O., Crampton, D., Lilly, S. J., Hammer, F., \& Tresse, L. 1995, ApJ, 455,60

Le Fèvre, O., Vettolani, G., Garilli, B., et al. 2005, A\&A, 439, 845

Lilly, S. J., Le Fevre, O., Hammer, F., \& Crampton, D. 1996, ApJ, 460, L1

Lilly, S. J., Le Fèvre, O., Renzini, A., et al. 2007, ApJS, 172, 70

Lilly, S. J., LeBrun, V., Maier, C., et al. 2009, ApJS, 184, 218

Madau, P., Pozzetti, L., \& Dickinson, M. 1998, ApJ, 498, 106

Margoniner, V. E., \& de Carvalho, R. R. 2000, AJ, 119, 1562

Margoniner, V. E., de Carvalho, R. R., Gal, R. R., \& Djorgovski, S. G. 2001, ApJ, 548, L143

Marinoni, C., Davis, M., Newman, J. A., \& Coil, A. L. 2002, ApJ, 580, 122

Martínez, H. J., Zandivarez, A., Domínguez, M., Merchán, M. E., \& Lambas, D. G. 2002, MNRAS, 333, L31

Mignoli, M., Zamorani, G., Scodeggio, M., et al. 2009, A\&A, 493, 39

Moore, B., Lake, G., Quinn, T., \& Stadel, J. 1999, MNRAS, 304, 465

Poggianti, B. M., Smail, I., Dressler, A., et al. 1999, ApJ, 518, 576

Poggianti, B. M., von der Linden, A., De Lucia, G., et al. 2006, ApJ, 642, 188

Popesso, P., Biviano, A., Romaniello, M., \& Böhringer, H. 2007, A\&A, 461, 411

Pozzetti, L., Bolzonella, M., Lamareille, F., et al. 2007, A\&A, 474, 443

Pozzetti, L., Bolzonella, M., Zucca, E., et al. 2009, A\&A, submitted [arXiv: 0907.5416]

Rakos, K. D., \& Schombert, J. M. 1995, ApJ, 439, 47

Roediger, E., \& Hensler, G. 2005, A\&A, 433, 875

Schiminovich, D., Ilbert, O., Arnouts, S., et al. 2005, ApJ, 619, L47
Scoville, N., Aussel, H., Brusa, M., et al. 2007, ApJS, 172, 1

Silverman, J. D., Kovač, K., Knobel, C., et al. 2009, ApJ, 695, 171

Smail, I., Edge, A. C., Ellis, R. S., \& Blandford, R. D. 1998, MNRAS, 293, 124

Springel, V. 2005, MNRAS, 364, 1105

Strateva, I., Ivezić, Ž., Knapp, G. R., et al. 2001, AJ, 122, 1861

Tanaka, M., Goto, T., Okamura, S., Shimasaku, K., \& Brinkmann, J. 2004, AJ, 128,2677

Tanaka, M., Finoguenov, A., Kodama, T., et al. 2008, A\&A, 489, 571

Tasca, L. A. M., Kneib, J.-P., Iovino, A., et al. 2009, A\&A, 503, 379

van Dokkum, P. G., Franx, M., Fabricant, D., Illingworth, G. D., \& Kelson, D. D. 2000, ApJ, 541, 95

Vergani, D., Zamorani, G., Lilly, S. J., et al. 2010, A\&A, 509, A42

Vollmer, B., Beck, R., Kenney, J. D. P., \& van Gorkom, J. H. 2004, AJ, 127, 3375

Voronoi, G. 1908, J. Reine Angew. Math., 134, 198

Wechsler, R. H., Bullock, J. S., Primack, J. R., Kravtsov, A. V., \& Dekel, A. 2002, ApJ, 568, 52

Weinmann, P. G., PP, M., GG, D., Illingworth, G. D., \& Kelson, D. D. 2000, ApJ, 541, 95

Wilman, D. J., Balogh, M. L., Bower, R. G., et al. 2005a, MNRAS, 358, 88 Wilman, D. J., Balogh, M. L., Bower, R. G., et al. 2005b, MNRAS, 358, 71 Zabludoff, A. I., \& Mulchaey, J. S. 1998, ApJ, 496, 39

Zucca, E., Bardelli, S., Bolzonella, M., et al. 2009, A\&A, 508, 1217

1 INAF - Osservatorio Astronomico di Brera, via Brera, 28, 20159 Milano, Italy e-mail: angela.iovino@brera.inaf.it

2 Laboratoire d'Astrophysique de Marseille, CNRS-Université d'AixMarseille, 38 rue Frederic Joliot Curie, 13388 Marseille, France

3 INAF - IASF Milano, via Bassini 15, 20133, Milano, Italy

${ }^{4}$ Institute of Astronomy, ETH Zurich, 8093, Zürich, Switzerland

5 INAF - Osservatorio Astronomico di Bologna, via Ranzani 1, 40127 Bologna, Italy

6 Laboratoire d'Astrophysique de Toulouse-Tarbes, Université de Toulouse, CNRS, 14 Avenue Edouard Belin, 31400 Toulouse, France

7 INAF - Osservatorio Astrofisico di Arcetri, Largo Enrico Fermi 5, 50125 Firenze, Italy

8 Max-Planck-Institut für extraterrestrische Physik, 84571 Garching b. Muenchen, 85748 , Germany

9 European Southern Observatory, Karl-Schwarzschild-Strasse 2, Garching b. Muenchen, 85748, Germany

10 Dipartimento di Astronomia, Università di Padova, vicolo Osservatorio 3, 35122 Padova, Italy

11 INAF - Osservatorio Astronomico di Torino, Strada Osservatorio 20, 10025 Pino Torinese, Torino, Italy

12 Dept. of Astronomy, University of Massachusetts, 710 North Pleasant Street, Amherst, MA 01003-9305, USA

13 Dipartimento di Astronomia, Universitá di Bologna, via Ranzani 1, 40127, Bologna, Italy

14 Physics Division, MS 50 R5004, Lawrence Berkeley National Laboratory, 1 Cyclotron Rd., Berkeley, CA 94720, USA

15 Space Telescope Science Institute, 3700 San Martin Drive, Baltimore, MD 21218, USA

16 Centre de Physique Theorique, Campus de Luminy, Case 907 13288 Marseille, France

17 Institut d'Astrophysique de Paris, UMR 7095 CNRS, Université Pierre et Marie Curie, 98bis boulevard Arago, 75014 Paris, France

18 Universitäts-Sternwarte, Scheinerstrasse 1, Munich 81679, Germany

19 Argelander-Institut für Astronomie, Auf dem Hügel 71, 53121 Bonn, Germany

20 INAF, Osservatorio di Roma via di Frascati, 33, 00040 Monte Porzio Catone, Italy

21 Department of Astronomy, Columbia University, 550 West 120th Street, New York, NY 10027, USA

22 California Institute of Technology, MC 105-24, 1200 East California Boulevard, Pasadena, CA 91125, USA 